\title{
Invisible Higgs decay in a supersymmetric inverse seesaw model with light sneutrino dark matter
}

\author{
Shankha Banerjee, ${ }^{a}$ P.S. Bhupal Dev, ${ }^{b}$ Subhadeep Mondal, ${ }^{c}$ \\ Biswarup Mukhopadhyaya ${ }^{a}$ and Sourov Roy ${ }^{c}$ \\ ${ }^{a}$ Regional Centre for Accelerator-based Particle Physics, Harish-Chandra Research Institute, \\ Chhatnag Road, Jhusi, Allahabad 211019, India \\ ${ }^{b}$ Consortium for Fundamental Physics, School of Physics and Astronomy, \\ University of Manchester, \\ Manchester, M13 9PL, U.K. \\ ${ }^{c}$ Department of Theoretical Physics, Indian Association for the Cultivation of Science, \\ $2 A$ E $2 B$ Raja S.C. Mullick Road, Kolkata 700032, India \\ E-mail: shankha@hri.res.in, Bhupal.Dev@hep.manchester.ac.uk, \\ tpsm2@iacs.res.in, biswarup@hri.res.in, tpsr@iacs.res.in
}

ABStract: Within the framework of a constrained Minimal Supersymmetric Standard Model (cMSSM) augmented by an MSSM singlet-pair sector to account for the non-zero neutrino masses by inverse seesaw mechanism, the lightest supersymmetric particle (LSP) can be a mixed sneutrino with mass as small as $50 \mathrm{GeV}$, satisfying all existing constraints, thus qualifying as a light dark matter candidate. We study the possibility of the lightest neutral Higgs boson in this model decaying invisibly into a pair of sneutrino LSPs, thereby giving rise to novel missing energy signatures at the LHC. We perform a two-parameter global analysis of the LHC Higgs data available till date to determine the optimal invisible Higgs branching fraction in this scenario, and obtain a $2 \sigma(1 \sigma)$ upper limit of $0.25(0.15)$. A detailed cut-based analysis is carried out thereafter, demonstrating the viability of our proposed signal vis-a-vis backgrounds at the LHC.

KEYWORDS: Supersymmetry Phenomenology

ARXiv EPRINT: 1306.2143 


\section{Contents}

1 Introduction 1

2 An overview of the model $\quad 3$

2.1 Fitting neutrino oscillation data 4

2.2 Sneutrino mass matrix 5

3 Invisible Higgs decay $\quad 6$

3.1 Light sneutrino DM 6

$\begin{array}{lll}3.2 & \text { The invisible decay width and current data } & 7\end{array}$

$\begin{array}{ll}3.3 & \text { Upper limit on the Dirac Yukawa coupling } \\ \end{array}$

$\begin{array}{ll}3.4 & \text { Some benchmark points } \\ \end{array}$

4 Collider analysis $\quad 13$

$\begin{array}{llr}4.1 & \text { Event generation } & 14\end{array}$

$\begin{array}{ll}4.2 & \text { The VBF channel } \\ \end{array}$

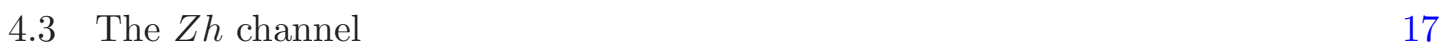

$\begin{array}{lll}5 & \text { Conclusion } & 19\end{array}$

$\begin{array}{ll}\text { A Higgs data sets } & 20\end{array}$

\section{Introduction}

After the recent discovery [1, 2] of a Higgs boson with mass around $125 \mathrm{GeV}$, a major goal is to establish whether it is 'the' Standard Model (SM) Higgs boson or a first glimpse of some Beyond Standard Model (BSM) physics at the LHC. A precise determination of the discovered Higgs boson characteristics will be crucial in resolving some of the outstanding issues of the SM, and in particular, understanding the mechanism of electroweak symmetry breaking and its relationship to the BSM. The experimental results so far [3-6] show no significant deviation from the SM Higgs sector expectations, and already put severe constraints on various new physics models (see, for instance, [7-15]). However, they still do not exclude the possibility of a non-standard Higgs boson.

A precise measurement of the total decay width of the Higgs boson $(h)$ through its line shape is very difficult at the LHC due to its tiny value: for the SM with $m_{h}=125 \mathrm{GeV}$, $\Gamma_{h}=4.07 \mathrm{MeV}[16]$. Hence, a better way to identify a non-standard Higgs boson is by studying its non-standard decay modes (for a review, see e.g., [17]). This is also crucial in case of a statistically significant discrepancy between the measured and SM expected Higgs signal strengths which could be due to either suppression or enhancement of the Higgs production cross section as well as its partial decay widths. 
A particularly interesting non-standard Higgs decay which is very sensitive to large BSM contributions is its invisible decay mode [18], since the SM invisible Higgs branching ratio (BR) is very small: $\mathrm{BR}\left(h \rightarrow Z Z^{*} \rightarrow 4 \nu\right) \simeq 0.001$ [19]. Dedicated searches for the Higgs decay into invisible final states were performed at the LEP [20], and no signal was found for Higgs mass up to $114.4 \mathrm{GeV}$. The LHC prospects of determining the invisible Higgs BR have been analyzed in refs. [21-30]. The current experimental limits for a $125 \mathrm{GeV}$ invisible Higgs BR are $<0.65$ from ATLAS [31] and $<0.75$ from CMS [32] at 95\% $\mathrm{CL}$ derived from the direct search $p p \rightarrow Z h \rightarrow \ell \ell \mathbb{E}_{T}$. Global fits to the existing LHC data provide a stronger constraint on $\mathrm{BR}_{\text {inv }}<0.28$ at 95\% CL [13] (for other recent global fits, see $[33,34])$.

From a phenomenological point of view, the compelling evidence for the existence of dark matter (DM) and its 'WIMP-miracle explanation' (for a review, see e.g., [35]) suggest that given suitable mass and unsuppressed coupling to the Higgs, the invisible decay to DM could be significant. In fact, this can occur in many well-motivated BSM scenarios, e.g., MSSM with neutralino DM [36-43], models with extended scalar sector [44-49], Majoron models [50-54], large extra dimension [55, 56], etc. The possibility of Higgs decaying to DM has gained renewed interest in view of the recent claims from some DM direct detection experiments such as DAMA/LIBRA [57], CoGeNT [58], CRESST-II [59], and more recently CDMS-II [60], in favor of a light DM in the mass range 5-50 GeV, with a large DM-nucleon scattering cross section of $10^{-5}-10^{-7} \mathrm{pb}$. This provides a strong motivation to examine the invisible Higgs decays in some BSM scenarios accommodating a light DM.

Due to various well-known theoretical reasons (see e.g., [61]), low-scale supersymmetry (SUSY) remains as one of the most attractive BSM scenarios, in spite of the null results from SUSY searches at the LHC so far $[62,63]$. In $R$-parity conserving SUSY models, the lightest supersymmetric particle (LSP), if electrically neutral, provides a natural WIMP DM candidate (for a review, see [64]). In the Minimal Supersymmetric Standard Model (MSSM), the lightest neutralino is the usual DM candidate, as the other viable candidate, namely, the scalar superpartner of the left-handed ( $\mathrm{LH}$ ) neutrino (the LH sneutrino), is strongly disfavored due to constraints from DM relic density and direct detection as well as the invisible decay width of the $Z$-boson $[65,66]$. However, in the minimal Supergravity (mSUGRA)/constrained MSSM (cMSSM) [67] with gaugino and sfermion mass unification, the recent LHC data disfavor a light neutralino mass below about $200 \mathrm{GeV}$, as demonstrated by the global fits [68-71], thus excluding the possibility of Higgs decaying to neutralino DM. ${ }^{1}$ For other implications of the recent experimental results for mSUGRA, see e.g., [76].

On the other hand, the neutrino oscillation data require at least two of the three SM neutrinos to have a tiny but non-zero mass (for a review, see e.g., [77]) which calls for some new physics beyond SM/MSSM. Thus, it would be interesting if a simple extension of the MSSM to explain the neutrino oscillation data can also accommodate a light DM candidate while satisfying all the existing experimental constraints. Such a scenario was recently studied in ref. [78] within the framework of cMSSM supplemented by a SM singlet-

\footnotetext{
${ }^{1}$ In a more general version of the MSSM it is still possible to have a light neutralino satisfying all the experimental constraints $[72,73]$ though these cases turn out to be highly fine-tuned (see e.g., [74, 75]).
} 
pair sector to explain the non-zero neutrino masses and mixing by a low-scale inverse seesaw mechanism $[79,80]$. It was shown that in contrast with the pure cMSSM scenario, this allows a light DM in the form of a mixed sneutrino with mass around $m_{h} / 2$, required to have a large annihilation rate via $s$-channel Higgs resonance.

Due to the large Yukawa couplings allowed in the model which are responsible for an efficient annihilation of the sneutrino DM, the lightest $C P$-even Higgs boson can have a large invisible branching ratio to sneutrino final states. This in turn leads to novel missing energy signatures at the LHC. Here we analyze this possibility in detail by performing a two-parameter global fit with the latest LHC Higgs data to determine the optimal invisible Higgs branching ratio allowed in this model, and find a $2 \sigma(1 \sigma)$ upper limit of $0.25(0.15)$. This in turn puts an upper limit of $\mathcal{O}(0.1)$ on the Dirac Yukawa coupling in the model. We further show that the model parameter space allowed by the invisible Higgs decay constraints can be completely ruled out in case of null results at the next generation DM direct detection experiments such as LUX and XENON1T. Finally, we select a few benchmark points satisfying all the experimental constraints, and carry out a detailed cutbased analysis, demonstrating the viability of our proposed signal in two Higgs production channels, namely, vector boson fusion (VBF) and associated production with $Z$, vis-avis SM backgrounds at $\sqrt{s}=14 \mathrm{TeV}$ LHC. We find that a signal significance of $3 \sigma$ can be achieved in the VBF channel with an integrated luminosity as low as $200 \mathrm{fb}^{-1}$, whereas in the $Z h$ channel it requires a luminosity of at least $600 \mathrm{fb}^{-1}$ for our chosen benchmark points.

The paper is organized in the following way. In section 2 we give a brief description of the model. In section 3, we scan the model parameter space to select a few benchmark points for a viable light sneutrino DM candidate. Then we perform a global $\chi^{2}$-analysis with the available LHC Higgs data to obtain the $1 \sigma$ and $2 \sigma$ allowed ranges of invisible Higgs branching fraction. In section 4 we present a collider analysis for the invisible Higgs decay signature at the LHC, focusing on two of its production channels, namely, VBF and $Z h$, for a few chosen benchmark points satisfying all the experimental constraints. Our conclusions are given in section 5. In the appendix, we list all the ATLAS and CMS Higgs data sets used in our global analysis.

\section{An overview of the model}

In the supersymmetric version of the inverse seesaw mechanism [79, 80], all the light neutrino masses can be generated at tree-level by adding three pairs of SM singlet superfields: $\hat{N}_{i}^{c}$ and $\hat{S}_{i}$ (with $i=1,2,3$ ) having lepton number -1 and +1 respectively. Thus the sneutrino LSP is in general a linear combination of the superpartners of the LH neutrino and the singlet fermions. Several embeddings of this set up have been discussed in the literature within the MSSM gauge group [81] as well as with extended gauge symmetries such as $\mathrm{SU}(2)_{L} \times \mathrm{SU}(2)_{R} \times \mathrm{U}(1)_{B-L}[82-84], \mathrm{SU}(2)_{L} \times \mathrm{U}(1)_{Y} \times \mathrm{U}(1)_{B-L}[85,86]$ and $\mathrm{SU}(2)_{L} \times \mathrm{U}(1)_{Y} \times \mathrm{U}(1)_{R}[87]$. In this paper, we choose to work within the MSSM gauge group and take a hybrid approach for the model parameters similar to that in refs. [78, 81] to find suitable benchmark points, i.e. a low energy input for the MSSM singlet fermion sector and for the lepton-number violating soft SUSY-breaking sector while a top-down 
approach for the MSSM sparticle spectrum with mSUGRA boundary conditions at the high scale without necessarily imposing any features of a specific Grand Unified Theory (GUT) framework. The mSUGRA boundary conditions for the MSSM sector enables us a direct comparison with the pure cMSSM case for its collider phenomenology [78]. The low-energy inputs for the singlet sector are chosen to satisfy all the low-energy constraints in the lepton sector. It is reasonable to choose them directly at the SUSY-breaking scale since the Renormalization Group (RG) running effects from the singlet sector on the pure cMSSM sector are expected to be small (as can be seen, for instance, from the RG equations in ref. [83] in the context of a particular $\mathrm{SO}(10)$ GUT model), and hence, it is equivalent to choosing a corresponding set of RG-evolved high-energy inputs, thus making our analysis independent of any specific GUT embedding. Henceforth, we will refer to this hybrid model generically as the Supersymmetric Inverse Seesaw Model (SISM).

The SISM superpotential is given by

$$
\mathcal{W}_{\mathrm{SISM}}=\mathcal{W}_{\mathrm{MSSM}}+\epsilon_{a b} y_{\nu}^{i j} \hat{L}_{i}^{a} \hat{H}_{u}^{b} \hat{N}_{j}^{c}+M_{R_{i j}} \hat{N}_{i}^{c} \hat{S}_{j}+\mu_{S_{i j}} \hat{S}_{i} \hat{S}_{j}
$$

$\mu_{S}$ being the only (tiny) source of lepton number violation in the superpotential. The soft SUSY-breaking Lagrangian is given by

$$
\begin{aligned}
\mathcal{L}_{\mathrm{SISM}}^{\text {soft }}= & \mathcal{L}_{\mathrm{MSSM}}^{\mathrm{soft}}-\left[m_{N}^{2} \widetilde{N}^{c^{\dagger}} \widetilde{N}^{c}+m_{S}^{2} \widetilde{S}^{\dagger} \widetilde{S}\right] \\
& -\left[\epsilon_{a b} A_{\nu}^{i j} \widetilde{L}_{i}^{a} \widetilde{N}_{j}^{c} H_{u}^{b}+B_{M_{R}}^{i j} \widetilde{N}_{i}^{c} \widetilde{S}_{j}+B_{\mu_{S}}^{i j} \widetilde{S}_{i} \widetilde{S}_{j}+\text { h.c. }\right]
\end{aligned}
$$

As a result of the LH neutrinos mixing with the singlet ones, the tree level neutrino mass matrix is $9 \times 9$ in the basis $\left\{\nu_{L}, N^{c}, S\right\}$ :

$$
\mathcal{M}_{\nu}=\left(\begin{array}{ccc}
\mathbf{0} & M_{D} & \mathbf{0} \\
M_{D}^{T} & \mathbf{0} & M_{R} \\
\mathbf{0} & M_{R}^{T} & \mu_{S}
\end{array}\right),
$$

where $M_{D}=v_{u} y_{\nu}$ is the Dirac neutrino mass matrix, $v_{u}=v \sin \beta$ being the vacuum expectation value (vev) of the $\hat{H}_{u}$ superfield in MSSM, with $v \simeq 174 \mathrm{GeV}$. In the limit $\left\|\mu_{S}\right\| \ll$ $\left\|M_{R}\right\|$ (where $\left.\|M\| \equiv \sqrt{\operatorname{Tr}\left(M^{\dagger} M\right)}\right)$, we can extract the $3 \times 3$ light neutrino mass matrix as

$$
M_{\nu}=\left[M_{D} M_{R}^{T^{-1}}\right] \mu_{S}\left[\left(M_{R}^{-1}\right) M_{D}^{T}\right]+\mathcal{O}\left(\mu_{S}^{2}\right) \equiv F \mu_{S} F^{T}+\mathcal{O}\left(\mu_{S}^{2}\right) .
$$

As can be seen from eq. (2.4), the smallness of neutrino mass now additionally depends on the small lepton-number violating parameter $\mu_{S}$ instead of just the smallness of the Dirac mass $M_{D}$ and/or heaviness of $M_{R}$ as in the canonical type-I seesaw case [88-92]. For $\mu_{S} \sim \mathcal{O}(\mathrm{keV})$, we can easily bring down $M_{R}$ to $\mathcal{O}(\mathrm{TeV})$ range even with comparatively large Dirac Yukawa couplings of $\mathcal{O}(0.1)$, thus leading to a rich collider phenomenology [78, 93-96] as well as observable lepton flavor violation (LFV) effects [82, 97-107].

\subsection{Fitting neutrino oscillation data}

The effective light neutrino mass matrix is usually diagonalized by the unitary PontecorvoMaki-Nakagawa-Sakata (PMNS) matrix. But due to its mixing with heavy neutrinos in 
the matrix structure of $\mathcal{M}_{\nu}$ in eq. (2.4), the light neutrino mixing matrix will receive additional non-unitary contributions. Thus, the full (non-unitary) light neutrino mixing matrix $\mathcal{U}$ diagonalizing the light neutrino mass matrix in eq. (2.4) has to be derived from the $9 \times 9$ unitary matrix $\mathcal{V}$ diagonalizing the full mass matrix given in eq. (2.3), i.e,

$$
\mathcal{V} \mathcal{M}_{\nu} \mathcal{V}^{T}=\operatorname{diag}\left(m_{i}, m_{R_{j}}\right), \quad(i=1,2,3 ; j=1,2, \ldots, 6),
$$

and by decomposing it into the blocks

$$
\mathcal{V}_{9 \times 9}=\left(\begin{array}{ll}
\mathcal{U}_{3 \times 3} & \mathcal{K}_{3 \times 6} \\
\mathcal{K}_{6 \times 3}^{\prime} & \mathcal{N}_{6 \times 6}
\end{array}\right)
$$

For $\left\|M_{D}\right\| \ll\left\|M_{R}\right\|$, it is sufficient to expand $\mathcal{U}$ up to leading order in $F=M_{D} M_{R}^{T^{-1}}$ :

$$
\mathcal{U} \simeq\left(\mathbf{1}-\frac{1}{2} F F^{\dagger}\right) U \equiv(\mathbf{1}-\eta) U
$$

where U denotes the unitary Pontecorvo-Maki-Nakagawa-Sakata (PMNS) matrix that diagonalizes the light neutrino mass matrix and $\eta=\frac{1}{2} F F^{\dagger}$ is a measure of the non-unitarity.

In order to satisfy the LFV constraints simultaneously with the sneutrino DM relic density constraint, we choose to work with diagonal $M_{R}$ and $M_{D}$, and accordingly fit $\mu_{S}$ to be consistent with the neutrino oscillation data. We use the following global fit values for the oscillation parameters [108]:

$$
\begin{aligned}
& \Delta m_{21}^{2}=(7.62 \pm 0.19) \times 10^{-5} \mathrm{eV}^{2}, \quad \Delta m_{31}^{2}=(2.53 \pm 0.09) \times 10^{-3} \mathrm{eV}^{2} \\
& \sin ^{2} \theta_{12}=0.320 \pm 0.016, \quad \sin ^{2} \theta_{23}=0.490 \pm 0.065, \quad \sin ^{2} \theta_{13}=0.026 \pm 0.004
\end{aligned}
$$

\subsection{Sneutrino mass matrix}

In the scalar sector, due to mixing between doublet and singlet sneutrinos we have an analogous $9 \times 9$ complex (or $18 \times 18$ real) sneutrino mass squared matrix. Assuming $C P$ conservation in the soft SUSY-breaking Lagrangian $(2.2),{ }^{2}$ we can decompose this mass matrix into two $9 \times 9$ real block-diagonal matrices corresponding to $C P$-even and $C P$-odd sneutrino states. The corresponding mass term in the Lagrangian looks like

$$
\mathcal{L}_{\tilde{\nu}}=\frac{1}{2}\left(\phi^{R}, \phi^{I}\right)\left(\begin{array}{cc}
\mathcal{M}_{+}^{2} & \mathbf{0} \\
\mathbf{0} & \mathcal{M}_{-}^{2}
\end{array}\right)\left(\begin{array}{c}
\phi^{R} \\
\phi^{I}
\end{array}\right),
$$

where $\phi^{R, I}=\left(\widetilde{\nu}_{L_{i}}^{R, I}, \widetilde{N}_{j}^{c^{R, I}}, \widetilde{S}_{k}^{R, I}\right)(i, j, k=1,2,3)$ and

$$
\mathcal{M}_{ \pm}^{2}=\left(\begin{array}{ccc}
m_{\tilde{L}}^{2}+M_{D} M_{D}^{T}+\frac{1}{2} m_{Z}^{2} \cos 2 \beta & \pm\left(v_{u} A_{\nu}-\mu M_{D} \cot \beta\right) & M_{D} M_{R} \\
\pm\left(v_{u} A_{\nu}-\mu M_{D} \cot \beta\right)^{T} & m_{N}^{2}+M_{R} M_{R}^{T}+M_{D}^{T} M_{D} & B_{M_{R}} \pm M_{R} \mu_{S} \\
M_{R}^{T} M_{D}^{T} & B_{M_{R}}^{T} \pm \mu_{S} M_{R}^{T} & m_{S}^{2}+\mu_{S}^{2}+M_{R}^{T} M_{R} \pm B_{\mu_{S}}
\end{array}\right)
$$

\footnotetext{
${ }^{2}$ The addition of extra $C P$ phases do not affect any of the collider aspects studied in this paper; hence they were taken to be zero for simplicity.
} 
where $m_{\tilde{L}}^{2}$ denote the soft SUSY-breaking mass squared term for $\mathrm{SU}(2)_{L}$-doublet sleptons. The real symmetric $C P$-even and $C P$-odd mass squared matrices $\mathcal{M}_{ \pm}^{2}$ can be diagonalized by $9 \times 9$ orthogonal matrices $\mathcal{G}_{ \pm}$as follows:

$$
\mathcal{G}_{ \pm} \mathcal{M}_{ \pm}^{2} \mathcal{G}_{ \pm}^{T}=\operatorname{diag}\left(m_{\widetilde{\nu}_{i}^{R, I}}^{2}\right) \quad(i=1,2, \cdots, 9)
$$

The corresponding eigenvalues of $\mathcal{M}_{ \pm}^{2}$ are almost degenerate in nature, with the degeneracy between $\widetilde{\nu}_{i}^{R}$ and $\widetilde{\nu}_{i}^{I}$ lifted only due to the small lepton number breaking parameter $\mu_{S}$. We will choose some benchmark points for which the lightest sneutrino mass eigenstate is the LSP, and will serve as a light DM candidate.

\section{Invisible Higgs decay}

Our goal in this section is to find the prospects of the lightest $C P$-even Higgs boson decaying into two light DM particles in the form of sneutrino LSP, thereby leading to a missing energy signal at the LHC. In the SISM being discussed here, we have 5 mSUGRA parameters $m_{0}, m_{1 / 2}, \tan \beta, A_{0}, \operatorname{sign}(\mu)$ at high scale and the additional inverse seesaw parameters $M_{D}, M_{R}, \mu_{S}, B_{\mu_{S}}$ and $B_{M_{R}}$ whose input values are chosen at the low scale. For simplicity, we have assumed these low-energy neutrino sector parameters to be diagonal (apart from $\mu_{s}$ whose structure is fixed by neutrino oscillation data) so that we can easily satisfy the LFV constraints. Also, the trilinear $A_{\nu}$ term in the soft SUSY-breaking Lagrangian which controls the Higgs BR to sneutrinos is taken to be $\left(A_{\nu}\right)_{i j}=A_{0}\left(y_{\nu}\right)_{i j}$. Note that we require a large $A_{0}$ in order to have a large radiative correction to the lightest $C P$-even Higgs mass as required by the LHC observation, whereas the Dirac Yukawa coupling $y_{\nu}$ is also required to be large in order to provide an efficient annihilation channel for the sneutrino LSP. These two seemingly uncorrelated effects inevitably lead to a large invisible BR for the Higgs in the SISM.

\subsection{Light sneutrino DM}

It was shown in [78] that the observed DM relic density for light sneutrino LSPs in the SISM is obtained by resonant enhancement of the annihilation cross section in the Higgs-mediated $s$-channel process: $\widetilde{\nu}_{\mathrm{LSP}} \widetilde{\nu}_{\mathrm{LSP}} \rightarrow f \bar{f}$ (where $f$ denotes the SM fermion). This is illustrated in figure 1 which was obtained by choosing the input parameters in a sample range

$$
\begin{aligned}
m_{0} & \in[0.1,2.5] \mathrm{TeV}, & m_{1 / 2} & \in[0.65,2.5] \mathrm{TeV}, \\
\operatorname{diag}\left(y_{\nu}\right) & \in[0.01,0.2], & \left(M_{R}\right)_{11} & \in[100,800] \mathrm{GeV},
\end{aligned}
$$

and for a fixed $\tan \beta=10, \operatorname{sign}(\mu)=+1,\left(M_{R}\right)_{22,33}=1 \mathrm{TeV}, B_{\mu_{S}}=10^{-4} \mathrm{GeV}^{2}$, and $B_{M_{R}}=10^{6} \mathrm{GeV}^{2}$. We have chosen the mSUGRA parameter ranges shown here keeping in mind the LHC exclusion limits on the cMSSM parameter space [62, 63]. The parameter scan was performed using SSP [109], with the SISM implemented in SARAH [110-112], and the sparticle spectrum was generated using SPheno [113, 114], while DM relic density was calculated using micrOMEGAs $[115,116]$. All the points shown in figure 1 are required to 


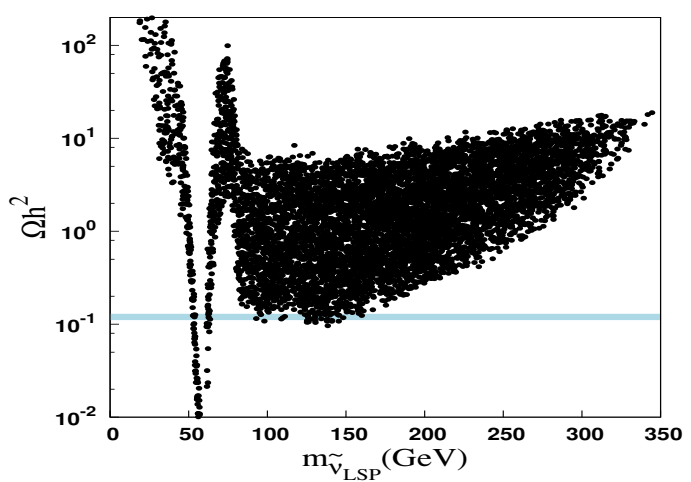

Figure 1. Sneutrino relic density as a function of the sneutrino LSP mass for our SISM input parameter scan. The horizontal shaded band shows the Planck $3 \sigma$ preferred range.

have the lightest $C P$-even Higgs mass in the range $125 \pm 2 \mathrm{GeV}$ to be consistent with the latest LHC Higgs data $[3,5]$. The horizontal blue band indicates the $3 \sigma$ preferred range from Planck data: $\Omega h^{2}=0.1199 \pm 0.0081$ [117]. It is clear that for the sneutrino LSP mass below $W$-boson mass, the observed DM relic density is obtained only near the Higgs-resonance region, thus requiring the sneutrino DM mass in the SISM to be around $m_{h} / 2$. The other possible resonance around $m_{Z} / 2$ is suppressed in this case due to small mixing between the $\mathrm{SU}(2)_{L}$-doublet and singlet neutrinos, as required to satisfy the $Z$-invisible decay width constraint from LEP [118]. Note that in figure 1, the observed relic density can also be satisfied for sneutrino LSP in the $80-200 \mathrm{GeV}$ mass range due to its large annihilation rate into $W W, Z Z$ and $h$ h final states. Since our main focus in this paper is on light sneutrino DM and Higgs invisible decay, we do not consider this mass range in our subsequent analysis.

The same interaction that leads to the Higgs-mediated $s$-channel annihilation of the sneutrino DM in our model also leads to a direct detection signal via $t$-channel Higgs exchange. In figure 2 we have plotted the spin-independent DM-nucleon scattering cross section predictions as a function of the sneutrino LSP mass for the corresponding points in figure 1. We also show the subset of points satisfying the relic density constraints. The solid line indicates the current limit from XENON100 data [119]. We also show the projected limits from XENON1T [120] and LUX [121] experiments. As evident from the plot, a few of the allowed points are already ruled out by the XENON100 data, while all of the low-mass points satisfying the relic density constraints can be ruled out by LUX and XENON1T projected limits in case of a null result.

From figures 1 and 2 we infer that it is indeed possible to have the lightest Higgs boson decaying into two sneutrino LSPs, while satisfying the DM relic density and direct detection constraints. We have also checked that all the points shown in figures 1 and 2 are well below the current indirect detection cross section limits from Fermi-LAT [122, 123].

\subsection{The invisible decay width and current data}

In order to ascertain how much invisible BR of the Higgs is allowed in our model, we perform a global analysis with all the LHC Higgs data available so far (see appendix). 


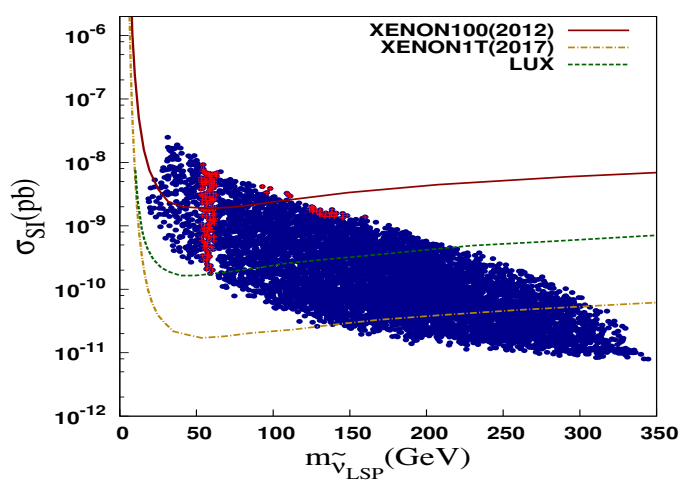

Figure 2. Spin-independent direct detection cross section as a function of the sneutrino LSP mass for our SISM parameter scan. The red $(+)$ points satisfy relic density $\lesssim 0.13$. The current experimental limit from XENON100 and the projected limits from LUX and XENON1T are also shown.

\begin{tabular}{|c|c|c|c|}
\hline Input parameter & BP1 & BP2 & BP3 \\
\hline$m_{0}(\mathrm{GeV})$ & 996.45 & 745.48 & 614.00 \\
$m_{1 / 2}(\mathrm{GeV})$ & 750.00 & 1014.17 & 1083.00 \\
$A_{0}(\mathrm{GeV})$ & -2858.00 & -2775.09 & -2600.00 \\
\hline
\end{tabular}

Table 1. The mSUGRA input parameters for three chosen benchmark points.

Since the neutrino sector parameters of the SISM do not affect the Higgs production or decay rates into the SM final states, and only affect its invisible decay into sneutrino final states, we can parametrize their effect in terms of a single free parameter, namely, the invisible BR $\varepsilon$ which relates the visible and invisible partial widths of the Higgs boson as

$$
\Gamma_{\mathrm{inv}}=\frac{\varepsilon}{1-\varepsilon} \sum \Gamma_{\mathrm{vis}} .
$$

For the MSSM sector of the SISM, we choose a few benchmark points (BPs) by fixing $m_{0}, m_{1 / 2}$ and $A_{0}$ as shown in table 1 , and vary the remaining parameter, namely, $\tan \beta$ to compute the low-energy SUSY spectrum using SPheno [113, 114]. The benchmark points given in table 1 were selected from the sample scan ranged over the values given in eq. (3.1) by requiring them to satisfy the constraints coming from higgs and squark-gluino mass bounds. We have fixed the sign of the MSSM $\mu$-parameter to be +1 throughout our analysis since $\mu<0$ is strongly disfavored by the muon anomalous magnetic moment as well as by the $B \rightarrow X_{s} \gamma$ branching ratio. Note that all the benchmark points shown in table 1 require an electroweak fine-tuning at the percent level, which is mandatory given the current LHC data (see e.g., [124]). For the trilinear term $A_{0}$, a large negative value is required to obtain the correct Higgs mass $\left(m_{h}=125 \pm 2 \mathrm{GeV}\right)$ for our choices of $m_{0}$ and $m_{1 / 2}$ (which are consistent with the general results from other mSUGRA parameter scans, e.g. [124]). We have checked that all our benchmark points lead to a stable electroweak vacuum and do not lead to charge- and/or color-breaking minima. 

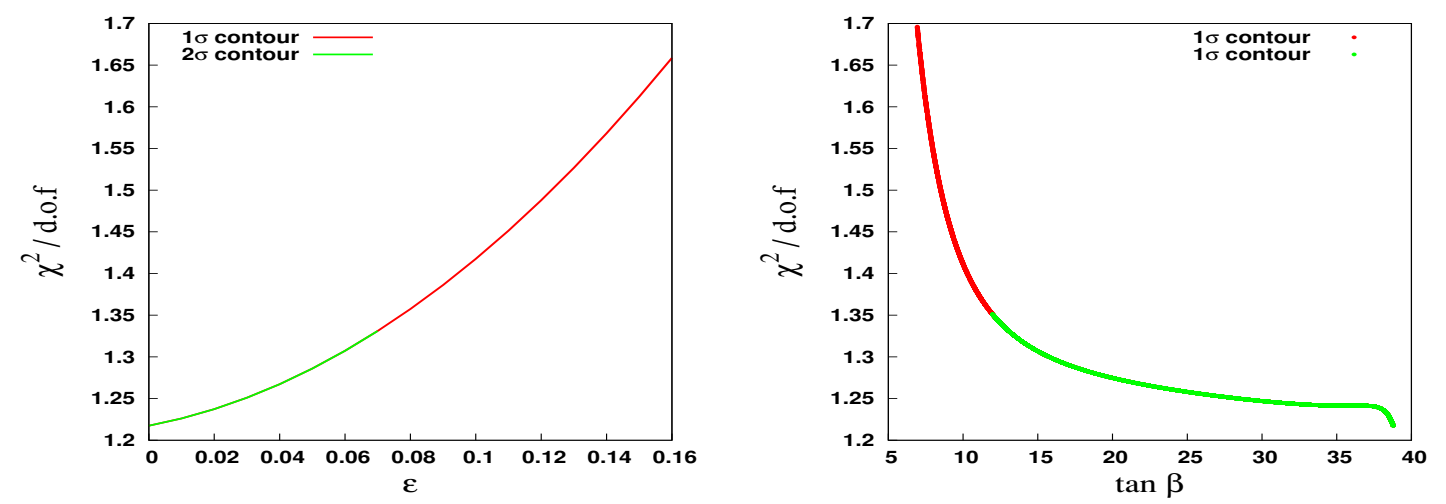

Figure 3. $1 \sigma$ and $2 \sigma$ contours for $\varepsilon$ and $\tan \beta$ from $\chi^{2}$ minimization obtained for BP1. The green line indicates $1 \sigma$ reach and the red line indicates $2 \sigma$ reach of the parameters.

For each combination of the high-scale parameters given in table 1, we perform a global analysis in the $\varepsilon$ - $\tan \beta$ plane using 10 data points in various Higgs decay channels from the published results of CMS and ATLAS, as listed in appendix. For each of the variables, with the other one marginalized, we compute the $\chi^{2}$ function, defined as

$$
\chi^{2}=\sum_{i} \frac{\left(\mu_{i}-\hat{\mu}_{i}\right)^{2}}{\left(\delta \hat{\mu}_{i}\right)^{2}}
$$

where $\mu_{i}$ 's are the Higgs signal strengths calculated from the model and are functions of the model parameters:

$$
\mu_{i}=R_{i}^{\text {prod }} \times \frac{R_{i}^{\text {decay }}}{R^{\text {width }}}
$$

Here $R_{i}$ 's are the ratios of the model predictions for the Higgs production cross sections and partial decay rates for various channels, and similarly $R$ is the ratio of the total width, with the corresponding SM expectations:

$$
R_{i}^{\text {prod }}=\frac{\left(\sigma_{i}^{\text {prod }}\right)_{\text {SISM }}}{\left(\sigma_{i}^{\text {prod }}\right)_{\mathrm{SM}}}, R_{i}^{\text {decay }}=\frac{\left(\Gamma_{i}^{\text {decay }}\right)_{\mathrm{SISM}}}{\left(\Gamma_{i}^{\text {decay }}\right)_{\mathrm{SM}}}, R^{\text {width }}=\frac{\left(\Gamma^{\text {width }}\right)_{\mathrm{SISM}}}{\left(\Gamma^{\text {width }}\right)_{\mathrm{SM}}},
$$

and $\hat{\mu_{i}}$ 's are the experimental best fit values of the signal strengths as listed in appendix, $\delta \hat{\mu}_{i}$ 's being their reported $1 \sigma$ uncertainty. When the reported uncertainties are asymmetric in nature, we consider the positive uncertainty for $\left(\mu_{i}-\hat{\mu}_{i}\right)>0$ and the negative one for $\left(\mu_{i}-\hat{\mu}_{i}\right)<0$.

We have varied $\tan \beta$ between 2 and 50 , and $\varepsilon$ between 0 and 0.7 . Note that large $\tan \beta \gtrsim 50$ is disfavored by the recent LHCb results on $B_{s} \rightarrow \mu^{+} \mu^{-}$, and very low $\tan \beta \lesssim 2$ are usually not considered due to radiative electroweak symmetry breaking arguments. The $1 \sigma$ and $2 \sigma$ contours for $\tan \beta$ and $\varepsilon$ for various 'snapshot' values of the high-scale parameters are presented in figures 3,4 and 5 . We have chosen the benchmark points for signal prediction, ensuring that we stay within $2 \sigma$ for both of the fitted parameters $(\varepsilon$ and $\tan \beta)$.

As manifested in these contour plots, the minimum $\chi^{2}$-value is obtained for $\varepsilon=0,0.037$ and 0.04 for BP1, BP2 and BP3 respectively and intermediate values of $\tan \beta$ around $30-40$ 

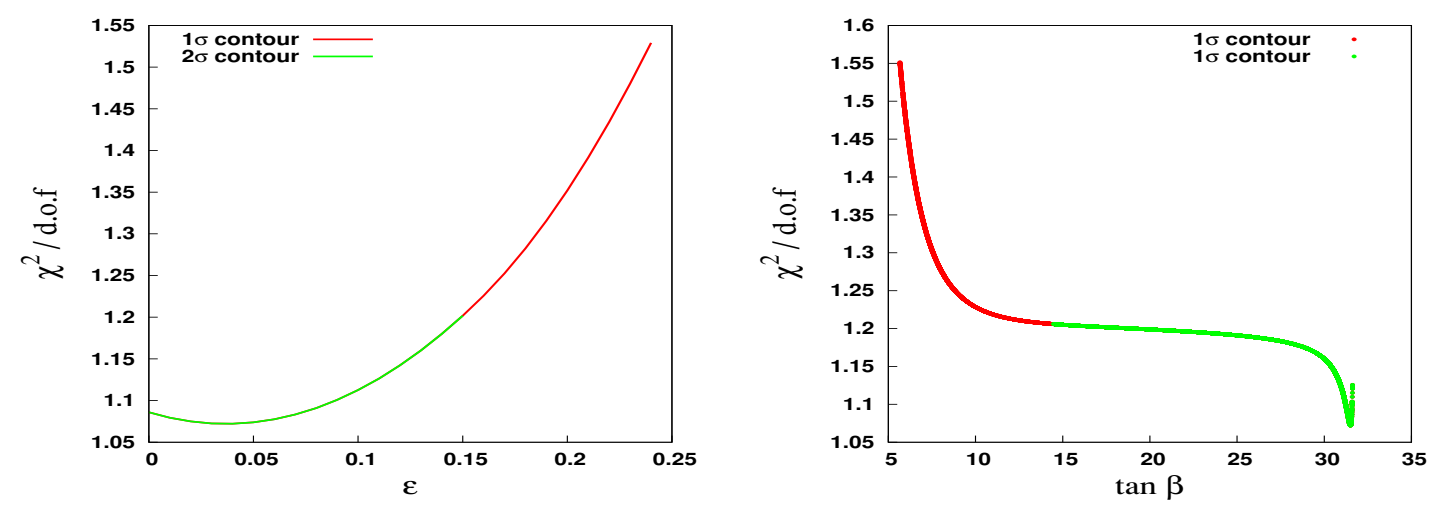

Figure 4. $1 \sigma$ and $2 \sigma$ contours for $\varepsilon$ and $\tan \beta$ from $\chi^{2}$ minimization obtained for BP2. The green line indicates $1 \sigma$ reach and the red line indicates $2 \sigma$ reach of the parameters.
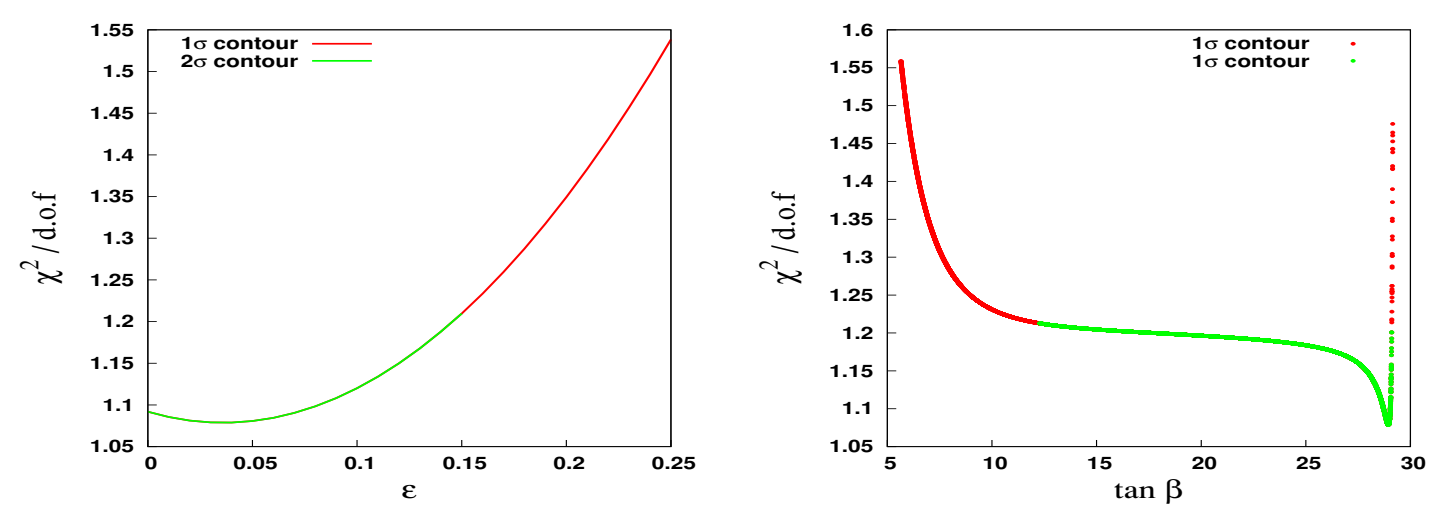

Figure 5. $1 \sigma$ and $2 \sigma$ contours for $\varepsilon$ and $\tan \beta$ from $\chi^{2}$ minimization obtained for BP3. The green line indicates $1 \sigma$ reach and the red line indicates $2 \sigma$ reach of the parameters.

\begin{tabular}{|c|c|c|c|c|c|c|}
\hline \multirow{2}{*}{ Parameter } & \multicolumn{2}{|c|}{ BP1 } & \multicolumn{2}{c|}{ BP2 } & \multicolumn{2}{c|}{ BP3 } \\
\cline { 2 - 7 } & $1 \sigma$ & $2 \sigma$ & $1 \sigma$ & $2 \sigma$ & $1 \sigma$ & $2 \sigma$ \\
\hline$\varepsilon$ & $<0.07$ & $<0.16$ & $<0.15$ & $<0.24$ & $<0.15$ & $<0.25$ \\
$\tan \beta$ & $12.0-38.8$ & $6.9-38.8$ & $14.5-31.6$ & $5.7-31.6$ & $12.3-29.1$ & $5.6-29.2$ \\
\hline
\end{tabular}

Table 2. The $1 \sigma$ and $2 \sigma$ limits on the invisible Higgs BR and the MSSM tan $\beta$ parameter obtained from the marginalized plots (figures $3-5$ ) for the chosen benchmark points in SISM.

for all the benchmark points. Also, there exists an upper limit on $\varepsilon$ to be consistent with the LHC Higgs data. The $68.27 \%\left(\Delta \chi^{2}=1\right)$ and $95.45 \%\left(\Delta \chi^{2}=4\right)$ CL limits derived from figures $3-5$ are summarized in table 2 , and also shown in figure 6 . These limits are comparable to those obtained in a recent model-independent global fit [13], and much stronger than the direct search limits from associated production of Higgs with $Z[31,32]$ as well as those derived from monojet searches [29]. 

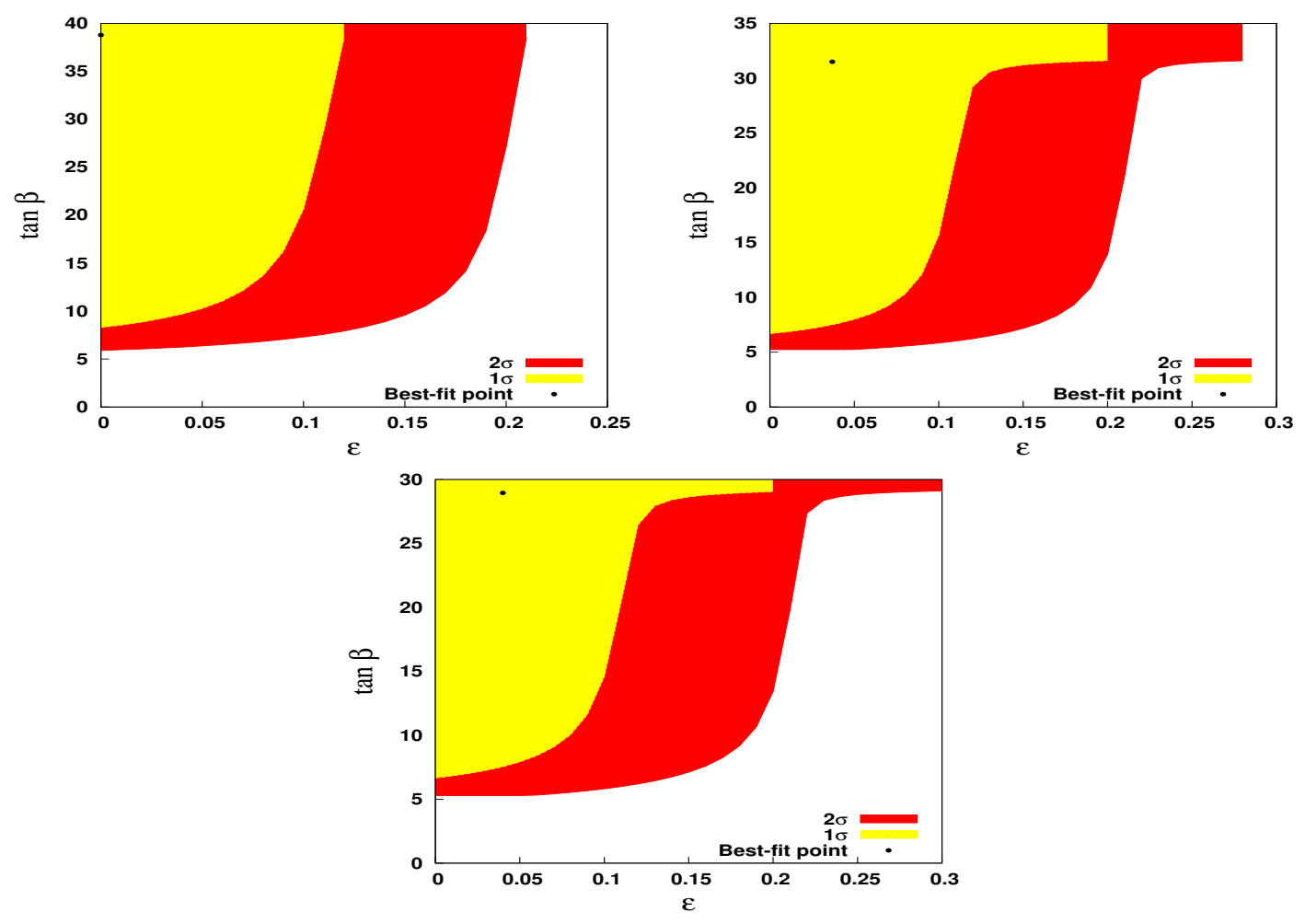

Figure 6. $1 \sigma$ and $2 \sigma$ allowed contours in the $\varepsilon-\tan \beta$ plane from global analysis of the Higgs data for the three benchmark points in our model. The black dots indicate the best fit values of $\tan \beta$ and $\varepsilon$ obtained from our analysis.

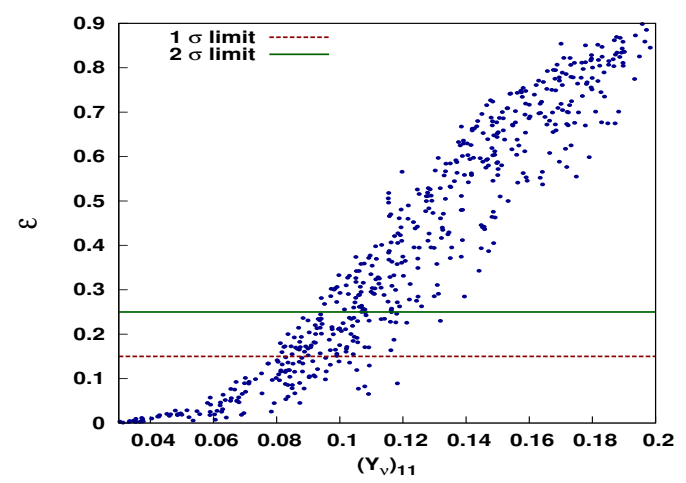

Figure 7. The invisible Higgs branching fraction as a function of the Dirac Yukawa coupling. The $1 \sigma$ and $2 \sigma$ upper limits on the invisible branching fraction derived earlier are also shown.

\subsection{Upper limit on the Dirac Yukawa coupling}

An upper limit on the invisible Higgs branching ratio, as derived in table 2 from a global analysis of the LHC Higgs data, will put an upper limit on the magnitude of the Dirac Yukawa coupling in the model. To illustrate this, we show in figure 7 the variation of the invisible Higgs branching fraction as a function of the Yukawa parameter, $\left(y_{\nu}\right)_{11}$. This plot 


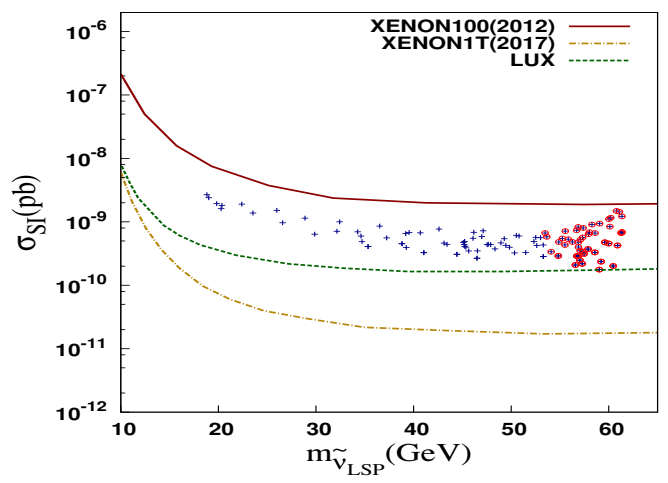

Figure 8. Spin-independent cross section as a function of the sneutrino LSP mass for the points satisfying the $2 \sigma$ upper limit on the invisible Higgs BR. The circled points also satisfy the relic density constraint.

is obtained for a fixed $A_{0}(\sim-2.8 \mathrm{TeV})$ and fixed $B_{M_{R}}$ and $B_{\mu_{S}}$ as given below eq. (3.1). However, other parameters are varied in the ranges mentioned in eq. (3.1). Note that, the invisible higgs BR is insensitive to other entries of $y_{\nu}$. We obtain a spread of the points as during the scan the Higgs mass fluctuates a little bit around its central value. Also the $M_{R}$ parameters vary which means that the LSP mass is not fixed at a particular value. It roughly varies between $20-62 \mathrm{GeV}$, and for most of the points, lie in the $30-62 \mathrm{GeV}$ range. As can be seen from the plot, the invisible Higgs branching fraction roughly grows with the Yukawa coupling in the kinematically allowed region. Thus an upper limit on the Dirac Yukawa coupling in the model follows from the upper limit on $\varepsilon$, as can be read off from the $1 \sigma$ and $2 \sigma$ lines in figure 7. Note that the upper limit of order of 0.10 on $y_{\nu}$ derived from this analysis is stronger than those derived from the Higgs visible decay [125] for a heavy neutrino mass larger than the Higgs mass. Comparable limits on $y_{\nu}$ in similar TeV scale seesaw models are obtained from charged-lepton flavour violating decays for the range of heavy neutrino masses we have considered here [126, 127].

The bound on $\varepsilon$ also constrains the allowed parameter space for the DM-nucleon elastic scattering cross section in this model. This is shown in figure 8 which is basically a zoomed-in version of figure 2 focusing on the light DM region and with only those points obeying the $2 \sigma(<25 \%)$ limit on $\varepsilon$. As can be seen from the plot, all these points are just below the current sensitivity of XENON100 experiment, but can be completely probed by the future experiments such as LUX and XENON1T.

\subsection{Some benchmark points}

In table 3 we present some benchmark values of the remaining model parameters not shown in table 1 as allowed by the invisible Higgs decay constraints. We have chosen the neutrino sector parameters to be diagonal, except for $\mu_{S}$ which was fixed by fitting the central global fit values of the neutrino oscillation parameters given in eq. (2.8). For illustration, we have assumed a normal hierarchy of neutrino masses with $m_{1}=10^{-5} \mathrm{eV}$ and the Dirac $C P$ phase $\delta=0$ in the PMNS matrix. It is clear from the choice of mSUGRA 


\begin{tabular}{|c|c|c|c|}
\hline Input parameter & BP1 & BP2 & BP3 \\
\hline $\tan \beta$ & 25 & 20 & 25 \\
$y_{\nu}$ & $(0.095,0.090,0.090)$ & $(0.074,0.064,0.064)$ & $(0.0701,0.010,0.010)$ \\
$M_{R}(\mathrm{GeV})$ & $(192.7,1000,1000)$ & $(679.16,1000,1000)$ & $(798,1000,1000)$ \\
$B_{\mu_{S}}\left(\mathrm{GeV}^{2}\right)$ & $10^{-4}$ & $10^{-4}$ & $10^{-4}$ \\
$B_{M_{R}}\left(\mathrm{GeV}^{2}\right)$ & $10^{6}$ & $10^{6}$ & $10^{6}$ \\
$\mu_{S}(\mathrm{eV})$ & $\left(\begin{array}{ccc}0.55 & 6.06 & 1.92 \\
6.06 & 107.86 & 87.97 \\
1.92 & 87.97 & 116.73\end{array}\right)$ & $\left(\begin{array}{ccc}11.25 & 38.73 & 12.29 \\
38.73 & 213.51 & 174.15 \\
12.29 & 174.15 & 231.08\end{array}\right)$ & $\left(\begin{array}{ccc}17.30 & 307.19 & 97.46 \\
307.19 & 8738.55 & 7127.59 \\
97.46 & 7127.59 & 9457.73\end{array}\right)$ \\
\hline
\end{tabular}

Table 3. Benchmark values of $\tan \beta$ and the low scale neutrino sector parameters for the chosen benchmark points in table 1.

parameters in table 1 that our low-energy MSSM particle spectrum is consistent with the current limits from direct SUSY searches $[62,63]$. We also calculate the other low-energy observables in the flavor sector using SPheno and in the DM sector using micrOMEGAS for the particle spectrum generated from SPheno using the input values shown in tables 1 and 3. These results, summarized in table 4, ensure that the chosen benchmark points are consistent with all the existing collider, cosmological and low energy constraints listed below (within their $3 \sigma$ allowed range, where applicable): (i) $m_{h}=125 \pm 2 \mathrm{GeV}[5,6]$, (ii) $\Omega h^{2}=0.1199 \pm 0.0027$ [117], (iii) $\sigma_{\mathrm{SI}}<5 \times 10^{-9} \mathrm{pb}$ for $m_{\mathrm{DM}} \simeq 50-60 \mathrm{GeV}$ [119], (iv) $\delta a_{\mu}=$ $(26.1 \pm 8.0) \times 10^{-10}[128]$ and $\delta a_{e}=(109 \pm 83) \times 10^{-14}[129],(\mathrm{v}) \operatorname{BR}\left(B \rightarrow X_{s} \gamma\right)=(3.21 \pm$ $0.33) \times 10^{-4}[130],(\mathrm{vi}) \operatorname{BR}\left(B_{s} \rightarrow \mu^{+} \mu^{-}\right)=\left(3.2_{-1.2}^{+1.5}\right) \times 10^{-9}[131]$, (vii) constraints from the LFV decays [132], and (viii) non-unitarity constraints in the neutrino sector $[133,134]$.

\section{Collider analysis}

The possibility of an invisible Higgs signature at the LHC has been explored both theoretically $[22,23,26-30]$ and experimentally [31, 32, 135]. These studies show that the most promising Higgs production channel for detecting an invisibly decaying Higgs is the vector boson fusion (VBF), and the next promising channel is its associated production with $Z$. In the VBF channel, Higgs is produced from vector bosons originated by radiation off two initial state quarks along with two jets, and subsequently decays into invisible final states: $p p \rightarrow q q h \rightarrow q q+\mathbb{E}_{T}$. Thus the final state consists of two jets widely separated in rapidity together with large missing transverse energy. In the $Z h$ associated production channel, the $Z$ decays into two oppositely charged leptons and the Higgs decays invisibly: $q \bar{q} \rightarrow Z+h \rightarrow \ell^{+} \ell^{-}+\mathbb{E}_{T}$. Note that the leptonic decay channel of $Z$ is known to be cleaner than its hadronic counterpart with $b$-jets. One can also look for an associated $W h$ production where $W$ decays leptonically to give rise to a $\ell+\mathbb{E}_{T}$ final state. However, the signal acceptance efficiency in this channel is found to be very small, and hence, the corresponding exclusion limit is much worse than that from the $Z h$ channel [136]. In addition to these channels, the dominant Higgs production channel at the LHC, namely, gluon-gluon fusion ( $\mathrm{ggF}$ ), can give rise to a monojet+large $\mathbb{E}_{T}$ signal with the jet coming from initial 


\begin{tabular}{|c|c|c|c|}
\hline Parameter & BP1 & BP2 & BP3 \\
\hline$m_{h}(\mathrm{GeV})$ & 124.69 & 125.79 & 125.78 \\
\hline$\Omega_{\mathrm{DM}} h^{2}$ & 0.114 & 0.122 & 0.112 \\
$\sigma_{\mathrm{SI}}(\mathrm{pb})$ & $3.38 \times 10^{-10}$ & $5.26 \times 10^{-10}$ & $5.56 \times 10^{-10}$ \\
\hline$\delta a_{\mu}$ & $3.1 \times 10^{-10}$ & $2.5 \times 10^{-10}$ & $3.4 \times 10^{-10}$ \\
$\delta a_{e}$ & $7.0 \times 10^{-15}$ & $5.7 \times 10^{-15}$ & $7.8 \times 10^{-15}$ \\
\hline $\mathrm{BR}\left(B \rightarrow X_{s} \gamma\right)$ & $2.9 \times 10^{-4}$ & $3.1 \times 10^{-4}$ & $3.1 \times 10^{-4}$ \\
$\mathrm{BR}\left(B_{s} \rightarrow \mu^{+} \mu^{-}\right)$ & $3.7 \times 10^{-9}$ & $3.5 \times 10^{-9}$ & $3.6 \times 10^{-9}$ \\
\hline $\mathrm{BR}(\mu \rightarrow e \gamma)$ & $5.2 \times 10^{-22}$ & $1.1 \times 10^{-22}$ & $3.5 \times 10^{-22}$ \\
$\mathrm{BR}(\tau \rightarrow e \gamma)$ & $9.8 \times 10^{-21}$ & $2.1 \times 10^{-21}$ & $6.6 \times 10^{-21}$ \\
$\operatorname{BR}(\tau \rightarrow \mu \gamma)$ & $1.6 \times 10^{-16}$ & $3.5 \times 10^{-17}$ & $1.1 \times 10^{-16}$ \\
$\operatorname{BR}(\mu \rightarrow 3 e)$ & $1.1 \times 10^{-22}$ & $8.9 \times 10^{-25}$ & $2.7 \times 10^{-24}$ \\
$\operatorname{BR}(\tau \rightarrow 3 e)$ & $6.8 \times 10^{-22}$ & $2.5 \times 10^{-23}$ & $7.7 \times 10^{-23}$ \\
$\operatorname{BR}(\tau \rightarrow 3 \mu)$ & $2.8 \times 10^{-16}$ & $3.0 \times 10^{-19}$ & $7.9 \times 10^{-19}$ \\
\hline$\left|\eta_{e e}\right|$ & $3.67 \times 10^{-3}$ & $1.79 \times 10^{-4}$ & $1.16 \times 10^{-4}$ \\
$\left|\eta_{\mu \mu}\right|$ & $1.22 \times 10^{-4}$ & $6.18 \times 10^{-5}$ & $1.51 \times 10^{-6}$ \\
$\left|\eta_{\tau \tau}\right|$ & $1.22 \times 10^{-4}$ & $6.18 \times 10^{-5}$ & $1.51 \times 10^{-6}$ \\
\hline
\end{tabular}

Table 4. The Higgs mass, relic density, spin-independent cross section, anomalous magnetic moments and the relevant low-energy flavor sector observables in the SISM for the three chosen BPs.

state radiation and Higgs decaying invisibly. But the QCD background for this process is too large, and moreover, it is hard to isolate the new physics effects only for the Higgs invisible decay since these effects could also show up in loops to modify the ggF production cross section. The $\sqrt{s}=7 \mathrm{TeV}$ search results in this channel $[137,138]$ were translated to a weak upper limit on $\varepsilon<0.4-0.6$ [29] depending on the jet $p_{T}$ threshold selection. Finally, the other relevant Higgs production channel, namely in association with top pairs, has a much smaller cross section [16], and involves complex final states which require a very sophisticated analysis. Therefore, we will focus on the VBF channel with 2 jets $+\mathbb{E}_{T}$ final states and the $Z h$ channel with $\ell^{+} \ell^{-}+\mathbb{E}_{T}$ final states for the collider analysis of invisible Higgs signature in our model. We show our analysis results for $\sqrt{s}=14 \mathrm{TeV}$ LHC.

\subsection{Event generation}

The SUSY particle spectrum and various decay branching fractions in our model have been calculated using SPheno $[113,114]$. The SLHA files are then fed to PYTHIA (version 6.409) [139] for event generation. The initial and final state radiation of quarks and gluons, multiple interactions, decay, hadronization, fragmentation and jet formation are implemented following the standard procedures in PYTHIA. The factorization and renormalization scales $\mu_{R}$ and $\mu_{F}$ respectively are both set at the parton-level center of mass energy 
$\sqrt{\hat{s}}$. We have used the CTEQ5L $[140,141]$ parton distribution functions in our analysis. The jets with $p_{T}>20 \mathrm{GeV}$ and $|\eta|<4.5$ have been constructed using the cone algorithm via PYCELL. To simulate detector effects, we take into account the smearing of jet energies by a Gaussian probability density function of width $\sigma(E) / E_{j}=\left(0.6 / \sqrt{E_{j}[\mathrm{GeV}]}\right)+0.03, E_{j}$ being the unsmeared jet energy [142].

Following are the selection cuts that we have used to find the final state leptons and jets:

- For final state electrons and muons we use $p_{T}>15 \mathrm{GeV}$ and $p_{T}>10 \mathrm{GeV}$ respectively. For both, we take $|\eta|<2.4$.

- Lepton-lepton separation $\Delta R_{\ell \ell}>0.2$, where $\Delta R=\sqrt{(\Delta \eta)^{2}+(\Delta \phi)^{2}}$.

- Lepton-jet separation $\Delta R_{\ell j}>0.4$.

- Scalar sum of $E_{T}$ deposits by hadrons within a cone of $\Delta R \leq 0.2$ around a lepton must be less than $0.2 p_{T}^{\ell}$ to ensure lepton isolation.

- Jet-jet separation $\Delta R_{j j}>0.4$.

Depending on the hadronic or leptonic signal final states, we use specialized selection criteria, as discussed below.

\subsection{The VBF channel}

In this case, the two leading high $p_{T}$ jets in the final state are produced in forward and backward directions with rapidities opposite in sign and widely separated. Also due to the invisible decay of the Higgs, one expects a large amount of missing energy. These features largely help to reduce the SM background. The dominant SM background for this signal can come from:

(i) $W+$ jets, where $W$ decays leptonically and the lepton escapes detection.

(ii) $Z+$ jets, where $Z$ decays into two neutrinos.

(iii) mismeasured QCD events giving fake missing energy.

The contributions from non-VBF processes, for instance, from hard QCD production of a single Higgs or a Higgs with associated quarks and gluons, must also be taken into account for the signal. Despite its poor efficiency to pass the background reducing cuts, due to its large production cross section the ggF channel can contribute $4-5 \%$ of the VBF signal $[143,144]$. The following cuts have been used to reduce the background:

- Absolute rapidity difference between the two leading jets, $\left|\eta_{j_{1}}-\eta_{j_{2}}\right|>4$.0. To ensure that the two jets are produced in forward and backward directions, we require $\eta_{j_{1}} \cdot \eta_{j_{2}}<0$.

- A jet veto with $p_{T}>40 \mathrm{GeV}$ in the central region since we don't expect any jets in the rapidity gap of the two jets for a pure VBF process. We discard jets with $|\eta|<2.5$ 


\begin{tabular}{|c|c|c|}
\hline Channel & Production cross section $(\mathrm{pb})$ & Cross section after cuts $(\mathrm{fb})$ \\
\hline BP1 (VBF) & 3.76 & 0.99 \\
BP1 (others) & 125.9 & 0.16 \\
BP2 (VBF) & 3.72 & 1.55 \\
BP2 (others) & 125.4 & 0.25 \\
BP3 (VBF) & 3.73 & 1.72 \\
BP3 (others) & 125.7 & 0.25 \\
\hline$W+$ n-jets & 56848.54 & 46.57 \\
$Z+$ n-jet & 10198.72 & 24.90 \\
\hline
\end{tabular}

Table 5. Final cross sections obtained for all the signal and SM background channels for a $14 \mathrm{TeV}$ LHC run. For the background channels, the cross sections in the 2nd column are those of the final states, i.e, $W$ and $Z$ decays into lepton-neutrino and two neutrino channels respectively. The cross sections in the 3rd column are the ones obtained after all the selection and background reduction cuts. n-jets corresponds to $0,1,2,3$ jets combined result.

- Invariant mass of the two leading jets, $M_{j j}>1.8 \mathrm{TeV}$.

- A $\mathbb{E}_{T}$ cut of $100 \mathrm{GeV}$.

The $\mathbb{E}_{T}$ and $M_{j j}$ cuts reduce the background efficiently, and also reduce the QCD contributions significantly. We note here that two additional cuts have been occasionally used in the literature for isolating events with invisible final states. These are $\Delta \phi\left(j, \mathbb{E}_{T}\right)$ and $\Delta \phi\left(j_{1}, j_{2}\right)$. We have checked that these cuts reduce the signal cross section to far too a level in our case. Therefore, we have dropped them and used the optimal set of event selection criteria mentioned above.

The cross sections for the signal corresponding to the benchmark points chosen earlier as well as dominant backgrounds coming from $W+n$-jets and $Z+n$-jets $(n=0,1,2,3)$ are shown in table 5. The background events were generated using Alpgen [145] at the partonic level and then passed to PYTHIA for showering. While interfacing, we have incorporated the MLM [146] prescription to match between the hard jets generated by Alpgen and the soft radiation jets generated by PYTHIA in order to avoid double counting. Since the background channels have huge inclusive cross sections, we generated at least $\sim 10^{7}$ unweighted events for all the channels in Alpgen in order to get proper convergence. For the signal cross section, we show the values obtained for VBF as well as for other hard processes $g g \rightarrow h, q \bar{q} \rightarrow g h, q g \rightarrow q h$ and $g g \rightarrow g h$. It is clear that, despite the large production cross section, contributions to the signal coming from channels other than VBF channel are very small after applying all the cuts. Also the SM backgrounds are hugely suppressed after all the cuts, optimized for a good signal significance, $\frac{S}{\sqrt{S+B}}$, where $S$ and $B$ stand for the signal and background strengths respectively. From table 5 , we find that for BP1 with the maximum ( $2 \sigma$ allowed) invisible branching ratio $\varepsilon_{\max }=0.16$ for the Higgs, we obtain a $3 \sigma$ signal significance at $500 \mathrm{fb}^{-1}$ whereas for BP2 and BP3 with $\varepsilon_{\max }=0.24$ and 0.25 respectively, we can obtain a $3 \sigma$ significance at $200 \mathrm{fb}^{-1}$. 


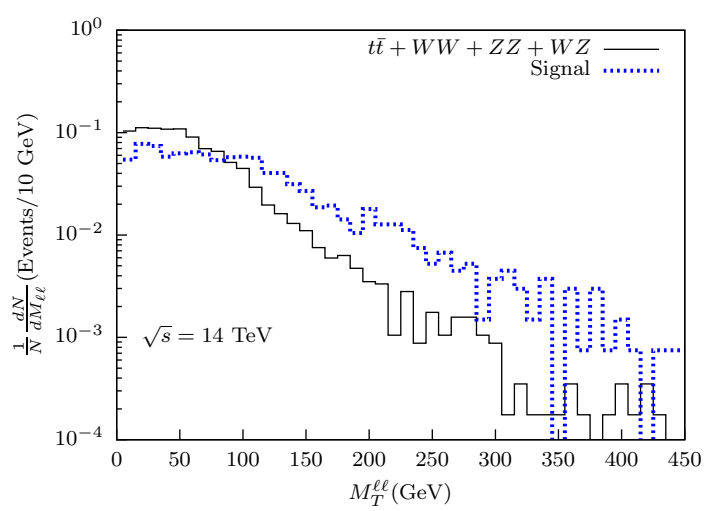

Figure 9. Normalized transverse mass distribution for the di-lepton system in the $Z h$ signal and combined SM background events at $14 \mathrm{TeV}$ LHC.

\subsection{The $Z h$ channel}

In this channel, we are interested in the leptonic decay of $Z$ leading to a same-flavor, opposite-sign dilepton plus large missing energy from the invisible decay of the Higgs. The dominant SM background in this case comes from:

(i) $W W$ production, where both the $W$ 's decay leptonically.

(ii) $W Z$ production, where $Z$ decays into two charged leptons and $W$ into a charged lepton and neutrino, and one charged lepton misses detection.

(iii) $Z Z$, where one $Z$ decays into two charged leptons and the other into two neutrinos.

(iv) $t \bar{t}$ production followed by $t \rightarrow W b$, where both the $W$ 's decay leptonically and the $b$-jets escape detection.

We use the following cuts to reduce the SM background:

- A jet veto with $p_{T}>20 \mathrm{GeV}$ and $|\eta|<4.5$ since the signal consists of no jets.

- Dilepton invariant mass $\left|M_{Z}-M_{\ell \bar{\ell}}\right|<10 \mathrm{GeV}$ since the two charged leptons in the final state come from $Z$-boson decay.

- Di-lepton transverse mass $M_{T}^{\ell \ell} \geq 150 \mathrm{GeV}$, where $M_{T}^{\ell \ell}=\sqrt{p_{T}^{\ell \ell} \mathbb{E}_{T}\left[1-\cos \phi\left(p_{T}^{\ell \ell}, \mathbb{E}_{T}\right)\right]}$. This is because the $Z$-boson and the Higgs are more likely produced back-to-back for the signal, thus leading to a harder transverse mass distribution for the di-lepton system, as can be seen from figure 9 .

- $\mathbb{E}_{T}>100 \mathrm{GeV}$ since the signal is expected to have a harder $\mathbb{E}_{T}$ distribution, as verified by figure 10 .

Table 6 shows the production cross sections and final cross sections after all the cuts for the signal corresponding to the chosen benchmark point as well as for the SM background. As can be seen from table 6 , this channel has a huge SM background which can easily 


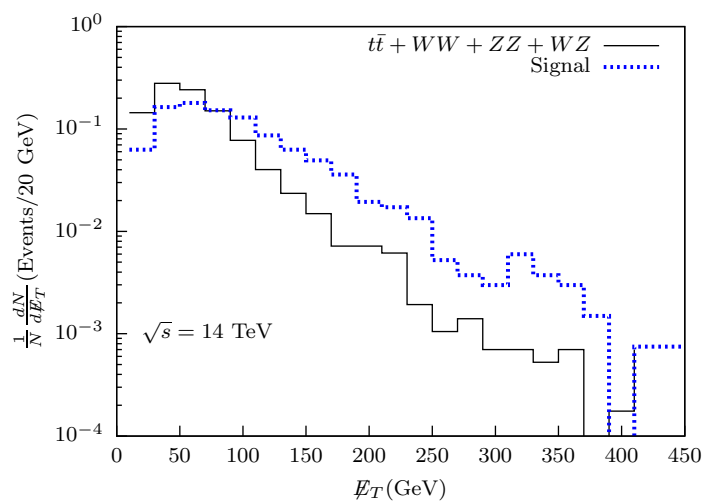

Figure 10. Missing transverse momentum distribution of the $Z h$ signal and combined SM background events at $14 \mathrm{TeV}$ LHC.

\begin{tabular}{|c|c|c|}
\hline Channel & Production cross section $(\mathrm{pb})$ & Cross section after cuts $(\mathrm{fb})$ \\
\hline BP1 & 0.53 & 0.35 \\
BP2 & 0.51 & 0.51 \\
BP3 & 0.51 & 0.52 \\
\hline$W W$ & 76.51 & 0.38 \\
$Z Z$ & 10.58 & 7.77 \\
$W Z$ & 28.95 & 8.83 \\
$t \bar{t}$ & 370.20 & 0.92 \\
\hline
\end{tabular}

Table 6. Cross sections obtained for all the signal and SM background channels for 14 TeV LHC. The 2nd column shows the production cross sections for various channels and the 3rd column after all the selection and background reduction cuts.

dominate over the signal events. The signal significance factor is quite low in this case for all the benchmark points. For BP2 and BP3 with a maximum invisible BR of Higgs $\varepsilon \sim 0.25$, the signal can achieve a significance of $3 \sigma$ only at $600 \mathrm{fb}^{-1}$ luminosity, whereas for BP1, to get such significance, we need to go beyond $1300 \mathrm{fb}^{-1}$ at $14 \mathrm{TeV}$ center of mass energy.

The reason for better LHC detection prospects for BP2 and BP3 compared to BP1 can be understood by comparing their corresponding particle spectra. The invisible higgs branching ratio $\varepsilon$ depends on the masses of the Higgs, LSP sneutrino and the Higgssneutrino-sneutrino coupling. Since the masses of the parent and daughter particles are almost identical for all the three cases, what makes the difference in the invisible decay width is the coupling which depends on the amount of mixing of the singlet sneutrinos with the left-handed ones. The singlet components dominate the lightest sneutrino mass eigenstates for all the three benchmarks because of the large $B_{M_{R}}$ term in the off-diagonal of the sneutrino mass matrix given by eq. (2.2). This parameter does not change for the three benchmark points and as a result, the right-handed components are not expected to vary much from BP1 to BP3. However, these components also depend on the matrices $m_{N}^{2}$, $m_{S}^{2}$ and $M_{R}$. Here $m_{N}^{2}$ and $m_{S}^{2}$ scale as $m_{0}^{2}$. Now from BP1 to BP3, $m_{0}$ keeps decreasing 
and $M_{R}$ keeps increasing. Hence the diagonal terms in eq. (2.2), although comparable, keep increasing slightly. This brings down the right-handed contribution in the lightest state by a very small amount from BP1 to BP3 (to be precise, the component comes down from 0.716 to 0.710$)$. On the other hand, as the absolute value of the trilinear term $A_{\nu}$ in eq. (2.2) decreases from BP1 to BP3, it brings down the left-component and increases the right-handed component. As a result of these competing effects, the left-component of the sneutrino LSP, and hence, the Higgs invisible decay width increases from BP1 to BP3, thus enhancing the LHC detection prospects.

Before concluding this section, we wish to emphasize an important distinction of our scenario from similar signals in the MSSM with a neutralino LSP which could otherwise obliterate the distinct collider signals of our model. As already pointed out in [78], the pure cMSSM case can be distinguished from the SISM case by studying the same-sign dilepton + jets $+\mathbb{E}_{T}$ signal which is enhanced in the SISM case. Also the SISM case has a much harder $\mathbb{E}_{T}$ tail compared to the cMSSM case which can be used as another distinguishing feature of our model. Finally, the "residual MSSM backgrounds" can be reduced/removed by studying the effective mass distribution of the events, defined as the scalar sum of the lepton and jet transverse momenta and missing transverse energy:

$$
M_{\text {eff }}=\sum\left|p_{T}^{\ell}\right|+\sum\left|p_{T}^{j}\right|+\mathbb{E}_{T} .
$$

Taking into account the current limits on the sparticle masses, the $M_{\text {eff }}$ distribution of events arising from sparticle production will be considerably harder in the pure MSSM case than in our case. Note that the cascade decays involving chargions can also be used to measure the mass of the sneutrino LSP at the LHC applying the $m_{T_{2}}$ endpoint technique [147].

\section{Conclusion}

We have shown that supplementing the cMSSM framework with inverse seesaw mechanism for neutrino masses can give rise to a light sneutrino DM candidate with mass around $50 \mathrm{GeV}$ while being consistent with all the existing collider, cosmological as well as lowenergy constraints. Such a light scalar DM also leads to the possibility of the lightest $C P$ even Higgs boson in the MSSM decaying invisibly into two such DM particles induced by a soft trilinear coupling. We have explored this possibility in details by performing a global $\chi^{2}$-analysis of all the available LHC Higgs data so far, and derive $2 \sigma(1 \sigma)$ upper limits of $0.25(0.15)$ on the invisible Higgs decay branching ratio in this scenario. These in turn put upper limits of order 0.1 on the Dirac Yukawa coupling in this model. We further show that the model parameter space allowed by the invisible Higgs decay branching ratio limits is fully accessible in the near future DM direct detection experiments such as LUX and XENON1T, and can be ruled out completely in case of a null result from these experiments. Finally, we have explored the prospects of the invisible Higgs decay signature at the $\sqrt{s}=14 \mathrm{TeV}$ LHC for a chosen set of benchmark points. We find that a signal significance of $3 \sigma$ can be achieved in the VBF channel with an integrated luminosity as low as $200 \mathrm{fb}^{-1}$, whereas in the $Z h$ channel, it requires a luminosity of at least $600 \mathrm{fb}^{-1}$ for our chosen benchmark points. 


\begin{tabular}{|c|c|c|}
\hline Channel & $\hat{\mu}$ & Experiment \\
\hline$h \rightarrow \gamma \gamma$ & $1.55_{-0.28}^{+0.33}$ & ATLAS [148] \\
& $0.78_{-0.26}^{+0.28}$ & CMS [149] \\
\hline$h \rightarrow Z Z^{*} \rightarrow 4 l$ & $1.43_{-0.35}^{+0.40}$ & ATLAS [148] \\
& $0.9_{-0.20}^{+0.30}$ & CMS [150] \\
\hline$h \rightarrow W W^{*} \rightarrow 2 l 2 \nu$ & $0.99_{-0.28}^{+0.31}$ & ATLAS [148] \\
& $0.80_{-0.20}^{+0.20}$ & CMS [151] \\
\hline$h \rightarrow b \bar{b}$ & $0.20_{-0.60}^{+0.70}$ & ATLAS (VH) [152] \\
& $1.00_{-0.50}^{+0.50}$ & CMS (VH) [153] \\
\hline$h \rightarrow \tau \bar{\tau}$ & $0.7_{-0.6}^{+0.7}$ & ATLAS [154] \\
& $1.10_{-0.4}^{+0.4}$ & CMS [155] \\
\hline
\end{tabular}

Table 7. Data set used in our analysis, with the values of $\hat{\mu}_{i}$ in various channels and their $1 \sigma$ uncertainties as reported by the ATLAS and CMS collaborations.

\section{Acknowledgments}

We are extremely thankful to the anonymous referee for carefully reading the whole manuscript, making many valuable suggestions, and checking some of the numerical results. We thank Sanjoy Biswas, Pushan Majumdar, Dipan Sengupta, and Florian Staub for useful correspondence, suggestions and comments at different stages of the work. SB and SM wish to thank Arindam Chatterjee and Satyanarayan Mukhopadhyay for some useful suggestions and comments. The work of PSBD is supported by the Lancaster-ManchesterSheffield Consortium for Fundamental Physics under STFC grant ST/J000418/1. SM acknowledges the hospitality of RECAPP, (HRI), Allahabad that led to this fruitful collaboration, and also wishes to thank the Department of Science and Technology, Government of India for a Senior Research Fellowship. S.B. and B.M. thank the Indian Association for the Cultivation of Science, Kolkata, for hospitality while this project was in progress. The work of SB and BM was partially supported by funding available from the Department of Atomic Energy, Government of India for the Regional Centre for Accelerator-based Particle Physics, Harish-Chandra Research Institute. Computational work for this study was partially carried out at the cluster computing facility in the Harish-Chandra Research Institute (http://cluster.hri.res.in/).

\section{A Higgs data sets}

In table 7 we list the latest Higgs data sets available from the combined $\sqrt{s}=7$ and $8 \mathrm{TeV}$ LHC run in five visible Higgs decay channels: $\gamma \gamma, Z Z^{*} \rightarrow 4 \ell, W W^{*} \rightarrow 2 \ell 2 \nu, b \bar{b}$ and $\tau \bar{\tau}$. For each channel, we show the experimental values of the signal strengths $\hat{\mu}_{i}$ together with its $1 \sigma$ uncertainty, as reported by the ATLAS and CMS collaborations [148-155]. 


\section{References}

[1] ATLAS collaboration, Observation of a new particle in the search for the Standard Model Higgs boson with the ATLAS detector at the LHC, Phys. Lett. B 716 (2012) 1 [arXiv:1207.7214] [INSPIRE].

[2] CMS collaboration, Observation of a new boson at a mass of $125 \mathrm{GeV}$ with the CMS experiment at the LHC, Phys. Lett. B 716 (2012) 30 [arXiv:1207.7235] [INSPIRE].

[3] ATLAS collaboration, Combined coupling measurements of the Higgs-like boson with the ATLAS detector using up to $25 \mathrm{fb}^{-1}$ of proton-proton collision data, ATLAS-CONF-2013-034, CERN, Geneva Switzerland (2013).

[4] ATLAS collaboration, Study of the spin of the new boson with up to $25 \mathrm{fb}^{-1}$ of ATLAS data, ATLAS-CONF-2013-040, CERN, Geneva Switzerland (2013).

[5] CMS collaboration, Combination of Standard Model Higgs boson searches and measurements of the properties of the new boson with a mass near $125 \mathrm{GeV}$, CMS-PAS-HIG-13-005, CERN, Geneva Switzerland (2013).

[6] ATLAS collaboration, Combined measurements of the mass and signal strength of the Higgs-like boson with the ATLAS detector using up to $25 \mathrm{fb}^{-1}$ of proton-proton collision data, ATLAS-CONF-2013-014, CERN, Geneva Switzerland (2013).

[7] T. Corbett, O. Eboli, J. Gonzalez-Fraile and M. Gonzalez-Garcia, Constraining anomalous Higgs interactions, Phys. Rev. D 86 (2012) 075013 [arXiv:1207.1344] [INSPIRE].

[8] T. Corbett, O. Eboli, J. Gonzalez-Fraile and M. Gonzalez-Garcia, Robust determination of the Higgs couplings: power to the data, Phys. Rev. D 87 (2013) 015022 [arXiv:1211.4580] [INSPIRE].

[9] S. Banerjee, S. Mukhopadhyay and B. Mukhopadhyaya, New Higgs interactions and recent data from the LHC and the Tevatron, JHEP 10 (2012) 062 [arXiv:1207.3588] [INSPIRE].

[10] G. Bélanger, B. Dumont, U. Ellwanger, J. Gunion and S. Kraml, Higgs couplings at the end of 2012, JHEP 02 (2013) 053 [arXiv:1212.5244] [INSPIRE].

[11] K. Cheung, J.S. Lee and P.-Y. Tseng, Higgs precision (Higgcision) era begins, JHEP 05 (2013) 134 [arXiv:1302.3794] [INSPIRE].

[12] A. Falkowski, F. Riva and A. Urbano, Higgs at last, arXiv:1303.1812 [INSPIRE].

[13] P.P. Giardino, K. Kannike, I. Masina, M. Raidal and A. Strumia, The universal Higgs fit, arXiv: 1303.3570 [INSPIRE].

[14] J. Ellis and T. You, Updated global analysis of Higgs couplings, JHEP 06 (2013) 103 [arXiv:1303.3879] [INSPIRE].

[15] A. Djouadi and G. Moreau, The couplings of the Higgs boson and its CP properties from fits of the signal strengths and their ratios at the $7+8 \mathrm{TeV} L H C$, arXiv:1303.6591 [INSPIRE].

[16] LHC Higgs Cross section Working Group collaboration, S. Dittmaier et al., Handbook of LHC Higgs cross sections: 1. Inclusive observables, arXiv:1101.0593 [INSPIRE].

[17] S. Chang, R. Dermisek, J.F. Gunion and N. Weiner, Nonstandard Higgs boson decays, Ann. Rev. Nucl. Part. Sci. 58 (2008) 75 [arXiv:0801.4554] [INSPIRE]. 
[18] R.E. Shrock and M. Suzuki, Invisible decays of Higgs bosons, Phys. Lett. B 110 (1982) 250 [INSPIRE].

[19] A. Denner, S. Heinemeyer, I. Puljak, D. Rebuzzi and M. Spira, Standard Model Higgs-boson branching ratios with uncertainties, Eur. Phys. J. C 71 (2011) 1753 [arXiv:1107.5909] [INSPIRE].

[20] DELPHI collaboration, J. Abdallah et al., Searches for invisibly decaying Higgs bosons with the DELPHI detector at LEP, Eur. Phys. J. C 32 (2004) 475 [hep-ex/0401022] [InSPIRE].

[21] J. Gunion, Detecting an invisibly decaying Higgs boson at a hadron supercollider, Phys. Rev. Lett. 72 (1994) 199 [hep-ph/9309216] [INSPIRE].

[22] S. Frederiksen, N. Johnson, G.L. Kane and J. Reid, Detecting invisible Higgs bosons at the CERN Large Hadron Collider, Phys. Rev. D 50 (1994) 4244 [InSPIRE].

[23] O.J. Eboli and D. Zeppenfeld, Observing an invisible Higgs boson, Phys. Lett. B 495 (2000) 147 [hep-ph/0009158] [INSPIRE].

[24] B.P. Kersevan, M. Malawski and E. Richter-Was, Prospects for observing an invisibly

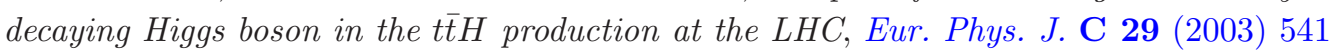
[hep-ph/0207014] [INSPIRE].

[25] K. Belotsky, D. Fargion, M. Khlopov, R. Konoplich and K. Shibaev, Invisible Higgs boson decay into massive neutrinos of fourth generation, Phys. Rev. D 68 (2003) 054027 [hep-ph/0210153] [INSPIRE].

[26] R. Godbole, M. Guchait, K. Mazumdar, S. Moretti and D. Roy, Search for 'invisible' Higgs signals at LHC via associated production with gauge bosons, Phys. Lett. B 571 (2003) 184 [hep-ph/0304137] [INSPIRE].

[27] H. Davoudiasl, T. Han and H.E. Logan, Discovering an invisibly decaying Higgs at hadron colliders, Phys. Rev. D 71 (2005) 115007 [hep-ph/0412269] [INSPIRE].

[28] S.-H. Zhu, Detecting an invisibly Higgs boson at Fermilab Tevatron and CERN LHC, Eur. Phys. J. C 47 (2006) 833 [hep-ph/0512055] [INSPIRE].

[29] Y. Bai, P. Draper and J. Shelton, Measuring the invisible Higgs width at the 7 and $8 \mathrm{TeV}$ LHC, JHEP 07 (2012) 192 [arXiv:1112.4496] [INSPIRE].

[30] D. Ghosh, R. Godbole, M. Guchait, K. Mohan and D. Sengupta, Looking for an invisible Higgs signal at the LHC, Phys. Lett. B 725 (2013) 344 [arXiv:1211.7015] [INSPIRE].

[31] ATLAS collaboration, Search for invisible decays of a Higgs boson produced in association with a $Z$ boson in ATLAS, ATLAS-CONF-2013-011, CERN, Geneva Switzerland (2013).

[32] CMS collaboration, Search for invisible Higgs produced in association with a $Z$ boson, CMS-PAS-HIG-13-018, CERN, Geneva Switzerland (2013).

[33] J.R. Espinosa, M. Muhlleitner, C. Grojean and M. Trott, Probing for invisible Higgs decays with global fits, JHEP 09 (2012) 126 [arXiv:1205.6790] [INSPIRE].

[34] G. Bélanger, B. Dumont, U. Ellwanger, J. Gunion and S. Kraml, Status of invisible Higgs decays, Phys. Lett. B 723 (2013) 340 [arXiv:1302.5694] [InSPIRE].

[35] G. Bertone, D. Hooper and J. Silk, Particle dark matter: evidence, candidates and constraints, Phys. Rept. 405 (2005) 279 [hep-ph/0404175] [INSPIRE].

[36] K. Griest and H.E. Haber, Invisible decays of Higgs bosons in supersymmetric models, Phys. Rev. D 37 (1988) 719 [INSPIRE]. 
[37] A. Djouadi, J. Kalinowski and P. Zerwas, Exploring the SUSY Higgs sector at $e^{+} e^{-}$linear colliders: a synopsis, Z. Phys. C 57 (1993) 569 [inSPIRE].

[38] G. Bélanger, F. Boudjema, A. Cottrant, R. Godbole and A. Semenov, The MSSM invisible Higgs in the light of dark matter and g-2, Phys. Lett. B 519 (2001) 93 [hep-ph/0106275] [INSPIRE].

[39] D. Albornoz Vasquez, G. Bélanger, R. Godbole and A. Pukhov, The Higgs boson in the MSSM in light of the LHC, Phys. Rev. D 85 (2012) 115013 [arXiv:1112.2200] [INSPIRE].

[40] A. Arbey, M. Battaglia and F. Mahmoudi, Constraints on the MSSM from the Higgs sector: a pMSSM study of Higgs searches, $B_{s}^{0} \rightarrow \mu^{+} \mu^{-}$and dark matter direct detection, Eur. Phys. J. C 72 (2012) 1906 [arXiv:1112.3032] [INSPIRE].

[41] N. Desai, B. Mukhopadhyaya and S. Niyogi, Constraints on invisible Higgs decay in MSSM in the light of diphoton rates from the LHC, arXiv:1202.5190 [INSPIRE].

[42] H.K. Dreiner, J.S. Kim and O. Lebedev, First LHC constraints on neutralinos, Phys. Lett. B 715 (2012) 199 [arXiv: 1206.3096] [INSPIRE].

[43] B. Ananthanarayan, J. Lahiri, P. Pandita and M. Patra, Invisible decays of the lightest Higgs boson in supersymmetric models, Phys. Rev. D 87 (2013) 115021 [arXiv:1306.1291] [INSPIRE].

[44] T. Binoth and J. van der Bij, Influence of strongly coupled, hidden scalars on Higgs signals, Z. Phys. C 75 (1997) 17 [hep-ph/9608245] [InSPIRE].

[45] C. Burgess, M. Pospelov and T. ter Veldhuis, The minimal model of nonbaryonic dark matter: a singlet scalar, Nucl. Phys. B 619 (2001) 709 [hep-ph/0011335] [INSPIRE].

[46] S. Andreas, C. Arina, T. Hambye, F.-S. Ling and M.H. Tytgat, A light scalar WIMP through the Higgs portal and CoGeNT, Phys. Rev. D 82 (2010) 043522 [arXiv:1003.2595] [INSPIRE].

[47] Y. Mambrini, Higgs searches and singlet scalar dark matter: combined constraints from XENON 100 and the LHC, Phys. Rev. D 84 (2011) 115017 [arXiv:1108.0671] [InSPIRE].

[48] M. Raidal and A. Strumia, Hints for a non-Standard Higgs boson from the LHC, Phys. Rev. D 84 (2011) 077701 [arXiv: 1108.4903] [INSPIRE].

[49] M. Pospelov and A. Ritz, Higgs decays to dark matter: beyond the minimal model, Phys. Rev. D 84 (2011) 113001 [arXiv:1109.4872] [INSPIRE].

[50] J. Romao, F. de Campos and J. Valle, New Higgs signatures in supersymmetry with spontaneous broken R parity, Phys. Lett. B 292 (1992) 329 [hep-ph/9207269] [INSPIRE].

[51] A.S. Joshipura and J. Valle, Invisible Higgs decays and neutrino physics, Nucl. Phys. B 397 (1993) 105 [inSPIRE].

[52] D. Choudhury and D. Roy, Signatures of an invisibly decaying Higgs particle at LHC, Phys. Lett. B 322 (1994) 368 [hep-ph/9312347] [INSPIRE].

[53] M. Hirsch, J. Romao, J. Valle and A. Villanova del Moral, Invisible Higgs boson decays in spontaneously broken R-parity, Phys. Rev. D 70 (2004) 073012 [hep-ph/0407269] [INSPIRE].

[54] K. Ghosh, B. Mukhopadhyaya and U. Sarkar, Signals of an invisibly decaying Higgs in a scalar dark matter scenario: a study for the Large Hadron Collider, Phys. Rev. D 84 (2011) 015017 [arXiv:1105.5837] [INSPIRE]. 
[55] A. Datta, K. Huitu, J. Laamanen and B. Mukhopadhyaya, Invisible Higgs in theories of large extra dimensions, Phys. Rev. D 70 (2004) 075003 [hep-ph/0404056] [INSPIRE].

[56] D. Dominici and J.F. Gunion, Invisible Higgs decays from Higgs graviscalar mixing, Phys. Rev. D 80 (2009) 115006 [arXiv:0902.1512] [inSPIRE].

[57] DAMA and LIBRA collaborations, R. Bernabei et al., New results from DAMA/LIBRA, Eur. Phys. J. C 67 (2010) 39 [arXiv:1002.1028] [InSPIRE].

[58] CoGeNT collaboration, C. Aalseth et al., Results from a search for light-mass dark matter with a P-type point contact germanium detector, Phys. Rev. Lett. 106 (2011) 131301 [arXiv: 1002.4703] [INSPIRE].

[59] G. Angloher et al., Results from $730 \mathrm{~kg}$ days of the CRESST-II dark matter search, Eur. Phys. J. C 72 (2012) 1971 [arXiv:1109.0702] [INSPIRE].

[60] CDMS collaboration, R. Agnese et al., Silicon detector dark matter results from the final exposure of CDMS II, Phys. Rev. Lett. accepted (2013) [arXiv:1304.4279] [INSPIRE].

[61] H. Baer and X. Tata, Weak scale supersymmetry, Cambridge University Press, Cambridge U.K. (2006).

[62] ATLAS experiment public results webpage, https://twiki.cern.ch/twiki/bin/view/AtlasPublic/SupersymmetryPublicResults.

[63] CMS experiment public results webpage, https://twiki.cern.ch/twiki/bin/view/CMSPublic/PhysicsResultsSUS.

[64] G. Jungman, M. Kamionkowski and K. Griest, Supersymmetric dark matter, Phys. Rept. 267 (1996) 195 [hep-ph/9506380] [INSPIRE].

[65] T. Falk, K.A. Olive and M. Srednicki, Heavy sneutrinos as dark matter, Phys. Lett. B 339 (1994) 248 [hep-ph/9409270] [INSPIRE].

[66] T. Hebbeker, Can the sneutrino be the lightest supersymmetric particle?, Phys. Lett. B 470 (1999) 259 [hep-ph/9910326] [INSPIRE].

[67] P. Nath, R. Arnowitt and A.H. Chamseddine, Applied $N=1$ supergravity, World Scientific, Singapore (1984).

[68] O. Buchmueller et al., The CMSSM and NUHM1 in light of $7 \mathrm{TeV} L H C, B_{s} \rightarrow \mu^{+} \mu^{-}$and XENON100 data, Eur. Phys. J. C 72 (2012) 2243 [arXiv:1207.7315] [InSPIRE].

[69] C. Strege et al., Global fits of the CMSSM and NUHM including the LHC Higgs discovery and new XENON100 constraints, JCAP 04 (2013) 013 [arXiv:1212.2636] [INSPIRE].

[70] M.E. Cabrera, J.A. Casas and R.R. de Austri, The health of SUSY after the Higgs discovery and the XENON100 data, arXiv:1212.4821 [INSPIRE].

[71] K. Kowalska, L. Roszkowski and E.M. Sessolo, Two ultimate tests of constrained supersymmetry, JHEP 06 (2013) 078 [arXiv: 1302.5956] [INSPIRE].

[72] D. Choudhury, H.K. Dreiner, P. Richardson and S. Sarkar, A supersymmetric solution to the KARMEN time anomaly, Phys. Rev. D 61 (2000) 095009 [hep-ph/9911365] [inSPIRE].

[73] H.K. Dreiner et al., Mass bounds on a very light neutralino, Eur. Phys. J. C 62 (2009) 547 [arXiv:0901.3485] [INSPIRE].

[74] P. Grothaus, M. Lindner and Y. Takanishi, Naturalness of neutralino dark matter, JHEP 07 (2013) 094 [arXiv: 1207.4434] [INSPIRE]. 
[75] C. Boehm, P.S.B. Dev, A. Mazumdar and E. Pukartas, Naturalness of light neutralino dark matter in pMSSM after LHC, XENON100 and Planck data, JHEP 06 (2013) 113 [arXiv: 1303.5386] [INSPIRE].

[76] P. Nath, SUGRA grand unification, LHC and dark matter, arXiv:1207.5501 [INSPIRE].

[77] M. Gonzalez-Garcia and M. Maltoni, Phenomenology with massive neutrinos, Phys. Rept. 460 (2008) 1 [arXiv:0704.1800] [InSPIRE].

[78] P. Bhupal Dev, S. Mondal, B. Mukhopadhyaya and S. Roy, Phenomenology of light sneutrino dark matter in cMSSM/mSUGRA with inverse seesaw, JHEP 09 (2012) 110 [arXiv:1207.6542] [INSPIRE].

[79] R. Mohapatra, Mechanism for understanding small neutrino mass in superstring theories, Phys. Rev. Lett. 56 (1986) 561 [INSPIRE].

[80] R. Mohapatra and J. Valle, Neutrino mass and baryon number nonconservation in superstring models, Phys. Rev. D 34 (1986) 1642 [INSPIRE].

[81] C. Arina, F. Bazzocchi, N. Fornengo, J. Romao and J. Valle, Minimal supergravity sneutrino dark matter and inverse seesaw neutrino masses,

Phys. Rev. Lett. 101 (2008) 161802 [arXiv:0806.3225] [INSPIRE].

[82] P.B. Dev and R. Mohapatra, TeV scale inverse seesaw in $\mathrm{SO}(10)$ and leptonic non-unitarity effects, Phys. Rev. D 81 (2010) 013001 [arXiv:0910.3924] [INSPIRE].

[83] P. Bhupal Dev and R. Mohapatra, Electroweak symmetry breaking and proton decay in SO(10) SUSY-GUT with TeV $W_{R}$, Phys. Rev. D 82 (2010) 035014 [arXiv:1003.6102] [INSPIRE].

[84] H. An, P.B. Dev, Y. Cai and R. Mohapatra, Sneutrino dark matter in gauged inverse seesaw models for neutrinos, Phys. Rev. Lett. 108 (2012) 081806 [arXiv:1110.1366] [INSPIRE].

[85] S. Khalil, H. Okada and T. Toma, Right-handed sneutrino dark matter in supersymmetric B-L model, JHEP 07 (2011) 026 [arXiv:1102.4249] [INSPIRE].

[86] L. Basso, O. Fischer and J. van der Bij, A natural $Z^{\prime}$ model with inverse seesaw and leptonic dark matter, Phys. Rev. D 87 (2013) 035015 [arXiv:1207.3250] [INSPIRE].

[87] V. De Romeri and M. Hirsch, Sneutrino dark matter in low-scale seesaw scenarios, JHEP 12 (2012) 106 [arXiv:1209.3891] [INSPIRE].

[88] P. Minkowski, $\mu \rightarrow$ er at a rate of one out of 1-billion muon decays?, Phys. Lett. B 67 (1977) 421 [INSPIRE].

[89] T. Yanagida, Horizontal symmetry and masses of neutrinos, in Workshop on unified theories, KEK report no. 79-18, KEK, Tsukuba Japan (1979), pg. 95 [Conf. Proc. C 7902131 (1979) 95] [INSPIRE].

[90] M. Gell-Mann, P. Ramond and R. Slansky, Complex spinors and unified theories, in Supergravity, D. Freedman et al. eds., North Holland, Amsterdam The Netherlands (1980), pg. 315 [Conf. Proc. C 790927 (1979) 315] [arXiv:1306.4669] [InSPIRE].

[91] R.N. Mohapatra and G. Senjanović, Neutrino mass and spontaneous parity violation, Phys. Rev. Lett. 44 (1980) 912 [INSPIRE].

[92] J. Schechter and J. Valle, Neutrino masses in $\mathrm{SU}(2) \times \mathrm{U}(1)$ theories, Phys. Rev. D 22 (1980) 2227 [INSPIRE]. 
[93] C.-Y. Chen and P.B. Dev, Multi-lepton collider signatures of heavy Dirac and Majorana neutrinos, Phys. Rev. D 85 (2012) 093018 [arXiv:1112.6419] [INSPIRE].

[94] S. Mondal, S. Biswas, P. Ghosh and S. Roy, Exploring novel correlations in trilepton channels at the LHC for the minimal supersymmetric inverse seesaw model, JHEP 05 (2012) 134 [arXiv:1201.1556] [INSPIRE].

[95] A. Das and N. Okada, Inverse seesaw neutrino signatures at LHC and ILC, arXiv:1207.3734 [INSPIRE].

[96] P. Bandyopadhyay, E.J. Chun, H. Okada and J.-C. Park, Higgs signatures in inverse seesaw model at the LHC, JHEP 01 (2013) 079 [arXiv:1209.4803] [INSPIRE].

[97] F. Deppisch and J. Valle, Enhanced lepton flavor violation in the supersymmetric inverse seesaw model, Phys. Rev. D 72 (2005) 036001 [hep-ph/0406040] [INSPIRE].

[98] F. Deppisch, T. Kosmas and J. Valle, Enhanced $\mu^{-}-e^{-}$conversion in nuclei in the inverse seesaw model, Nucl. Phys. B 752 (2006) 80 [hep-ph/0512360] [INSPIRE].

[99] M. Hirsch, T. Kernreiter, J. Romao and A. Villanova del Moral, Minimal supersymmetric inverse seesaw: neutrino masses, lepton flavour violation and LHC phenomenology, JHEP 01 (2010) 103 [arXiv:0910.2435] [INSPIRE].

[100] W. Abdallah, A. Awad, S. Khalil and H. Okada, Muon anomalous magnetic moment and $\mu \rightarrow$ er in B-L model with inverse seesaw, Eur. Phys. J. C 72 (2012) 2108 [arXiv: 1105.1047] [INSPIRE].

[101] A. Abada, D. Das and C. Weiland, Enhanced Higgs mediated lepton flavour violating processes in the supersymmetric inverse seesaw model, JHEP 03 (2012) 100 [arXiv:1111.5836] [INSPIRE].

[102] M. Hirsch, F. Staub and A. Vicente, Enhancing $l_{i} \rightarrow 3 l_{j}$ with the $Z^{0}$-penguin, Phys. Rev. D 85 (2012) 113013 [arXiv:1202.1825] [INSPIRE].

[103] A. Abada, D. Das, A. Vicente and C. Weiland, Enhancing lepton flavour violation in the supersymmetric inverse seesaw beyond the dipole contribution, JHEP 09 (2012) 015 [arXiv:1206.6497] [INSPIRE].

[104] M. Malinsky, T. Ohlsson and H. Zhang, Non-unitarity effects in a realistic low-scale seesaw model, Phys. Rev. D 79 (2009) 073009 [arXiv: 0903.1961] [INSPIRE].

[105] M. Malinsky, T. Ohlsson, Z.-Z. Xing and H. Zhang, Non-unitary neutrino mixing and CP-violation in the minimal inverse seesaw model, Phys. Lett. B 679 (2009) 242 [arXiv:0905.2889] [INSPIRE].

[106] R. Lal Awasthi and M.K. Parida, Inverse seesaw mechanism in nonsupersymmetric SO(10), proton lifetime, nonunitarity effects and a low-mass $Z^{\prime}$ boson, Phys. Rev. D 86 (2012) 093004 [arXiv:1112.1826] [INSPIRE].

[107] R.L. Awasthi, M. Parida and S. Patra, Neutrino masses, dominant neutrinoless double beta decay and observable lepton flavor violation in left-right models and $\mathrm{SO}(10)$ grand unification with low mass $W_{R}, Z_{R}$ bosons, JHEP 08 (2013) 122 [arXiv:1302.0672] [INSPIRE].

[108] D. Forero, M. Tortola and J. Valle, Global status of neutrino oscillation parameters after Neutrino-2012, Phys. Rev. D 86 (2012) 073012 [arXiv:1205.4018] [INSPIRE]. 
[109] F. Staub, T. Ohl, W. Porod and C. Speckner, A tool box for implementing supersymmetric models, Comput. Phys. Commun. 183 (2012) 2165 [arXiv:1109.5147] [InSPIRE].

[110] F. Staub, SARAH, arXiv:0806.0538 [INSPIRE].

[111] F. Staub, From superpotential to model files for FeynArts and CalcHep/CompHEP, Comput. Phys. Commun. 181 (2010) 1077 [arXiv:0909.2863] [INSPIRE].

[112] F. Staub, Automatic calculation of supersymmetric renormalization group equations and self energies, Comput. Phys. Commun. 182 (2011) 808 [arXiv: 1002.0840] [INSPIRE].

[113] W. Porod, SPheno, a program for calculating supersymmetric spectra, SUSY particle decays and SUSY particle production at $e^{+} e^{-}$colliders, Comput. Phys. Commun. 153 (2003) 275 [hep-ph/0301101] [INSPIRE].

[114] W. Porod and F. Staub, SPheno 3.1: extensions including flavour, CP-phases and models beyond the MSSM, Comput. Phys. Commun. 183 (2012) 2458 [arXiv:1104.1573] [INSPIRE].

[115] G. Bélanger, F. Boudjema, A. Pukhov and A. Semenov, MicrOMEGAs 2.0: a program to calculate the relic density of dark matter in a generic model,

Comput. Phys. Commun. 176 (2007) 367 [hep-ph/0607059] [INSPIRE].

[116] G. Bélanger, F. Boudjema, A. Pukhov and A. Semenov, Dark matter direct detection rate in a generic model with MicrOMEGAs 2.2, Comput. Phys. Commun. 180 (2009) 747 [arXiv:0803.2360] [INSPIRE].

[117] Planck collaboration, P. Ade et al., Planck 2013 results. XVI. Cosmological parameters, arXiv: 1303.5076 [INSPIRE].

[118] The LEP Electroweak Working Group webpage, http://lepewwg.web.cern.ch/LEPEWWG/.

[119] XENON100 collaboration, E. Aprile et al., Dark matter results from 225 live days of XENON100 data, Phys. Rev. Lett. 109 (2012) 181301 [arXiv:1207.5988] [INSPIRE].

[120] XENON1T collaboration, E. Aprile, The XENON1T dark matter search experiment, arXiv: 1206.6288 [INSPIRE].

[121] LUX collaboration, D. Akerib et al., The Large Underground Xenon (LUX) experiment, Nucl. Instrum. Meth. A 704 (2013) 111 [arXiv:1211.3788] [INSPIRE].

[122] Fermi-LAT collaboration, M. Ackermann et al., Constraining dark matter models from a combined analysis of milky way satellites with the Fermi Large Area Telescope, Phys. Rev. Lett. 107 (2011) 241302 [arXiv:1108.3546] [INSPIRE].

[123] LAT collaboration, M. Ackermann et al., Fermi LAT search for dark matter in gamma-ray lines and the inclusive photon spectrum, Phys. Rev. D 86 (2012) 022002 [arXiv:1205.2739] [INSPIRE].

[124] H. Baer et al., Post-LHC7 fine-tuning in the mSUGRA/CMSSM model with a $125 \mathrm{GeV}$ Higgs boson, Phys. Rev. D 87 (2013) 035017 [arXiv:1210.3019] [INSPIRE].

[125] P. Bhupal Dev, R. Franceschini and R. Mohapatra, Bounds on TeV seesaw models from LHC Higgs data, Phys. Rev. D 86 (2012) 093010 [arXiv:1207.2756] [INSPIRE].

[126] A. Ibarra, E. Molinaro and S. Petcov, Low energy signatures of the TeV scale see-saw mechanism, Phys. Rev. D 84 (2011) 013005 [arXiv:1103.6217] [INSPIRE]. 
[127] D. Dinh, A. Ibarra, E. Molinaro and S. Petcov, The $\mu-e$ conversion in nuclei, $\mu \rightarrow e \gamma$, $\mu \rightarrow 3 e$ decays and TeV scale see-saw scenarios of neutrino mass generation, JHEP 08 (2012) 125 [Erratum ibid. 09 (2013) 023] [arXiv: 1205.4671] [INSPIRE].

[128] K. Hagiwara, R. Liao, A.D. Martin, D. Nomura and T. Teubner, $(g-2)_{\mu}$ and $\alpha\left(M_{Z}^{2}\right)$ re-evaluated using new precise data, J. Phys. G 38 (2011) 085003 [arXiv:1105.3149] [INSPIRE].

[129] T. Aoyama, M. Hayakawa, T. Kinoshita and M. Nio, Tenth-order QED contribution to the electron $g-2$ and an improved value of the fine structure constant,

Phys. Rev. Lett. 109 (2012) 111807 [arXiv:1205.5368] [inSPIRE].

[130] BaBAR collaboration, J. Lees et al., Precision measurement of the $B \rightarrow X_{s} \gamma$ photon energy spectrum, branching fraction and direct $C P$ asymmetry $A_{C P}\left(B \rightarrow X_{s+d} \gamma\right)$,

Phys. Rev. Lett. 109 (2012) 191801 [arXiv:1207.2690] [inSPIRE].

[131] LHCb collaboration, First evidence for the decay $B_{s}^{0} \rightarrow \mu^{+} \mu^{-}$, Phys. Rev. Lett. 110 (2013) 021801 [arXiv: 1211.2674] [inSPIRE].

[132] Particle Data Group collaboration, J. Beringer et al., Review of particle physics (RPP), Phys. Rev. D 86 (2012) 010001 [inSPIRE].

[133] A. Abada, C. Biggio, F. Bonnet, M. Gavela and T. Hambye, Low energy effects of neutrino masses, JHEP 12 (2007) 061 [arXiv:0707.4058] [INSPIRE].

[134] S. Antusch, J.P. Baumann and E. Fernandez-Martinez, Non-standard neutrino interactions with matter from physics beyond the Standard Model, Nucl. Phys. B 810 (2009) 369 [arXiv:0807.1003] [INSPIRE].

[135] S. Bansal, K. Mazumdar and J.B. Singh, Search for invisibly decaying Higgs boson at Large Hadron Collider, Pramana 74 (2010) 231 [INSPIRE].

[136] P. Gagnon, Invisible Higgs boson decays in the $Z H$ and $W H$ channels, ATL-PHYS-PUB-2005-011, CERN, Geneva Switzerland (2005).

[137] ATLAS collaboration, Search for new phenomena in monojet plus missing transverse momentum final states using $1 \mathrm{fb}^{-1}$ of pp collisions at $\sqrt{s}=7 \mathrm{TeV}$ with the ATLAS detector, ATLAS-CONF-2011-096, CERN, Geneva Switzerland (2011).

[138] CMS collaboration, Search for new physics with a monojet and missing transverse energy in pp collisions at $\sqrt{s}=7 \mathrm{TeV}$, CMS-PAS-EXO-11-059, CERN, Geneva Switzerland (2011).

[139] T. Sjöstrand, S. Mrenna and P.Z. Skands, PYTHIA 6.4 physics and manual, JHEP 05 (2006) 026 [hep-ph/0603175] [INSPIRE].

[140] CTEQ collaboration, H. Lai et al., Global QCD analysis of parton structure of the nucleon: CTEQ5 parton distributions, Eur. Phys. J. C 12 (2000) 375 [hep-ph/9903282] [INSPIRE].

[141] J. Pumplin et al., New generation of parton distributions with uncertainties from global QCD analysis, JHEP 07 (2002) 012 [hep-ph/0201195] [INSPIRE].

[142] A.J. Barr and C. Gwenlan, The race for supersymmetry: using $m_{T 2}$ for discovery, Phys. Rev. D 80 (2009) 074007 [arXiv:0907.2713] [INSPIRE].

[143] V. Del Duca, W. Kilgore, C. Oleari, C. Schmidt and D. Zeppenfeld, Gluon fusion contributions to $H+2$ jet production, Nucl. Phys. B 616 (2001) 367 [hep-ph/0108030] [INSPIRE]. 
[144] A. Nikitenko and M.L. Vazquez Acosta, Monte Carlo study of $g g \rightarrow H+$ jets contribution to vector boson fusion Higgs production at the LHC, arXiv:0705.3585 [INSPIRE].

[145] M.L. Mangano, M. Moretti, F. Piccinini, R. Pittau and A.D. Polosa, ALPGEN, a generator for hard multiparton processes in hadronic collisions, JHEP 07 (2003) 001 [hep-ph/0206293] [INSPIRE].

[146] M.L. Mangano, Merging multijet matrix elements and shower evolution in hadronic collisions, talk at Lund University, http://cern.ch/ mlm/talks/lund-alpgen.pdf, Lund Sweden (2004).

[147] G. Bélanger, S. Kraml and A. Lessa, Light sneutrino dark matter at the LHC, JHEP 07 (2011) 083 [arXiv: 1105.4878] [INSPIRE].

[148] ATLAS collaboration, Measurements of Higgs boson production and couplings in diboson final states with the ATLAS detector at the LHC, Phys. Lett. B 726 (2013) 88 [arXiv: 1307.1427] [INSPIRE].

[149] CMS collaboration, Updated measurements of the Higgs boson at $125 \mathrm{GeV}$ in the two photon decay channel, CMS-PAS-HIG-13-001, CERN, Geneva Switzerland (2013).

[150] CMS collaboration, Properties of the Higgs-like boson in the decay $H \rightarrow Z Z \rightarrow 4 \ell$ in pp collisions at $\sqrt{s}=7$ and $8 \mathrm{TeV}$, CMS-PAS-HIG-13-002, CERN, Geneva Switzerland (2013).

[151] CMS collaboration, Evidence for a particle decaying to $W^{+} W^{-}$in the fully leptonic final state in a Standard Model Higgs boson search in pp collisions at the LHC, CMS-PAS-HIG-13-003, CERN, Geneva Switzerland (2013).

[152] ATLAS collaboration, Search for the bb decay of the Standard Model Higgs boson in associated W/ZH production with the ATLAS detector, ATLAS-CONF-2013-079, CERN, Geneva Switzerland (2013).

[153] CMS collaboration, Search for the Standard Model Higgs boson produced in association with $W$ or $Z$ bosons, and decaying to bottom quarks for LHCp 2013, CMS-PAS-HIG-13-012, CERN, Geneva Switzerland (2013).

[154] ATLAS collaboration, Search for the Standard Model Higgs boson in $H \rightarrow \tau \tau$ decays in proton-proton collisions with the ATLAS detector, ATLAS-CONF-2012-160, CERN, Geneva Switzerland (2012).

[155] CMS collaboration, Search for the Standard Model Higgs boson decaying to $\tau$ pairs in proton-proton collisions at $\sqrt{s}=7$ and $8 \mathrm{TeV}$, CMS-PAS-HIG-13-004, CERN, Geneva Switzerland (2013). 